\title{
Implicit-correction-based immersed boundary-lattice Boltzmann method with two relaxation times
}

\author{
Takeshi Seta* \\ Graduate School of Science and Engineering for Research, \\ University of Toyama, Toyama 930-8555, Japan
}

Rojas Molina Roberto Carlos, Kosuke Hayashi, and Akio Tomiyama
Graduate School of Engineering, Kobe University, Hyogo 657-8501, Japan

(Dated: January 29, 2014)

\begin{abstract}
In the present paper, we verify the effectiveness of the two-relaxation-time (TRT) collision operator in reducing boundary slip computed by the immersed boundary-lattice Boltzmann method (IB-LBM). In the linear collision operator of the TRT, we decompose the distribution function into symmetric and antisymmetric components and define the relaxation parameters for each part. The Chapman-Enskog expansion indicates that one relaxation time for the symmetric component is related to the kinematic viscosity. Rigorous analysis of the symmetric shear flows reveals that the relaxation time for the antisymmetric part controls the velocity gradient, the boundary velocity, and the boundary slip velocity computed by the IB-LBM. Simulation of the symmetric shear flows, the symmetric Poiseuille flows, and the cylindrical Couette flows indicates that the profiles of the numerical velocity calculated by the TRT collision operator under the IB-LBM framework exactly agree with those of the multi-relaxation time (MRT). The TRT is as effective in removing the boundary slip as the MRT. We demonstrate analytically and numerically that the error of the boundary velocity is caused by the smoothing technique using the delta function used in the interpolation method. In the simulation of the flow past a circular cylinder, the IB-LBM based on the implicit correction method with the TRT succeeds in preventing the flow penetration through the solid surface as well as unphysical velocity distortion. The drag coefficient, the wake length, and the separation points calculated by the present IB-LBM agree well with previous studies at Re $=$ 10,20 , and 40 .
\end{abstract}

PACS numbers: 47.11.-j, 02.60.Lj, 02.60.-x, 83.50.Rp

\section{INTRODUCTION}

The immersed boundary method (IBM) calculates fluid dynamics on an Eulerian grid and the particle motion on a Lagrangian grid in order to simulate the dynamics of solid particles in an incompressible viscous fluid [1]. In the lattice Boltzmann method (LBM), the Navier-Stokes equations are solved by the motions of the distribution functions streaming along discrete lattices based on the uniform Cartesian mesh [2]. Since the Cartesian mesh is used for fluid dynamics in both methods, the IBM is applied to the LBM with the efficiency in terms of the computational effort and memory requirement. Feng and Michaelides first proposed the immersed boundary-lattice Boltzmann method (IB-LBM), which treats a body force density as a spring force of a deformable boundary with a high stiffness [3]. Such a body force density is distributed into the Eulerian nodes of the grid to calculate interaction between the fluid and particles. A variety of IB-LBMs based on the momentum and energy conservation have been proposed for computing the force or heat balance between a solid wall and a fluid [4-8].

Le et al. proved that the numerical errors in the velocity and the velocity gradient at the boundary, which are

\footnotetext{
*seta@eng.u-toyama.ac.jp
}

referred to collectively as boundary slip, increase with the relaxation time $\tau$ [9]. In order to reduce boundary slip, we proposed an IB-LBM using a two-relaxation-time (TRT) model for the collision operator $[10,11]$. In the TRT model, we decompose the distribution function into symmetric and antisymmetric components. The relaxation time for the symmetric part of the velocity distribution function is used to determine the kinematic viscosity in the Navier-Stokes equations, whereas that for the antisymmetric part is used to improve the numerical accuracy. Ginzburg et al. extended the TRT model to the anisotropic advection dispersion equation and investigated the effect of a specific combination of two relaxation parameters on the accuracy by ChapmanEnskog analysis and Fourier analysis. Using von Neumann stability analysis, Ginzburg et al. proved that the TRT model is more stable than the single relaxation time (SRT) collision model, especially in the advectiondominant region [12].

Although two- and three-dimensional simulations of particle motion in an incompressible viscous fluid demonstrated that the IB-LBM with the TRT reduced the boundary slip, theoretical proofs were not given in Reference [11]. Lu et al. numerically and analytically demonstrated that the use of multi-relaxation time (MRT) [13] solved the problem of boundary slip in the IB-LBM [14]. Although more than one relaxation time in the collision operator removes the boundary slip of the IB-LBM, 
one problem remains, i.e., the disagreement between the boundary velocity $u_{j_{0}}$ on the Eulerian node and the desired velocity $u^{d}$ [14].

In the present study, we apply an error analysis proposed by Le and Zhang [9] and He et al. [15] to the IBLBM in order to compare the accuracy of the TRT model to that of the MRT model. In order to investigate the disagreement between $u_{j_{0}}$ and $u^{d}$, we examine the delta function used in the velocity and force interpolations between the Lagrangian nodes and the Eulerian nodes in the IB-LBM. A comparative study on the simulations of the flow past a circular cylinder calculated by various IBLBMs will clarify the influence of the boundary slip and the streamline penetration on the drag coefficient, the wake length, and the separation points.

\section{IMMERSED BOUNDARY-LATTICE BOLTZMANN METHOD}

\section{A. Lattice Boltzmann method}

The LBM for the incompressible Navier-Stokes equations uses the following kinetic equations for the distribution function $f_{k}$ :

$$
\begin{array}{r}
f_{k}\left(\mathbf{x}+\mathbf{e}_{k} \delta_{t}, t+\delta_{t}\right)=f_{k}(\mathbf{x}, t) \\
-\frac{f_{k}(\mathbf{x}, t)-f_{k}^{(e q)}(\mathbf{x}, t)}{\tau}+\delta_{t} F_{k}(\mathbf{x}, t),
\end{array}
$$

where $\mathbf{e}_{k}$ is the particle discrete velocity, $\delta_{t}$ is the time step, and $\tau$ is the relaxation time. The Bhatnagar-GrossKrook (BGK) single relaxation time (SRT) approximation is used for the collision matrix.

The fluid density and velocity are written in terms of the distribution function as follows:

$$
\rho=\sum_{k} f_{k}, \quad \mathbf{u}=\frac{1}{\rho} \sum_{k} f_{k} \mathbf{e}_{k} .
$$

The pressure is calculated by $p=c_{s}^{2} \rho$. The speed of sound $c_{s}$ is $c / \sqrt{3}$, where $c$ is the lattice velocity magnitude. The D2Q9 model is used in the present study. The discrete velocities are defined as

$$
\mathbf{e}_{k}= \begin{cases}(0,0), & k=0, \\ c\left(\cos \left(\frac{\pi(k-1)}{2}\right), \sin \left(\frac{\pi(k-1)}{2}\right)\right), & k=1-4,(3) \\ \sqrt{2} c\left(\cos \left(\frac{\pi(2 k-9)}{4}\right), \sin \left(\frac{\pi(2 k-9)}{4}\right)\right), & k=5-8 .\end{cases}
$$

The equilibrium distribution function for the D2Q9 model is given by

$$
f_{k}^{(e q)}=\omega_{k} \rho\left[1+\frac{3 \mathbf{e}_{k} \cdot \mathbf{u}}{c^{2}}+\frac{9\left(\mathbf{e}_{k} \cdot \mathbf{u}\right)^{2}}{2 c^{4}}-\frac{3 \mathbf{u} \cdot \mathbf{u}}{2 c^{2}}\right],
$$

where $\omega_{k}$ are the weight coefficients, i.e., $\omega_{0}=4 / 9$, $\omega_{1-4}=1 / 9$, and $\omega_{5-8}=1 / 36$.

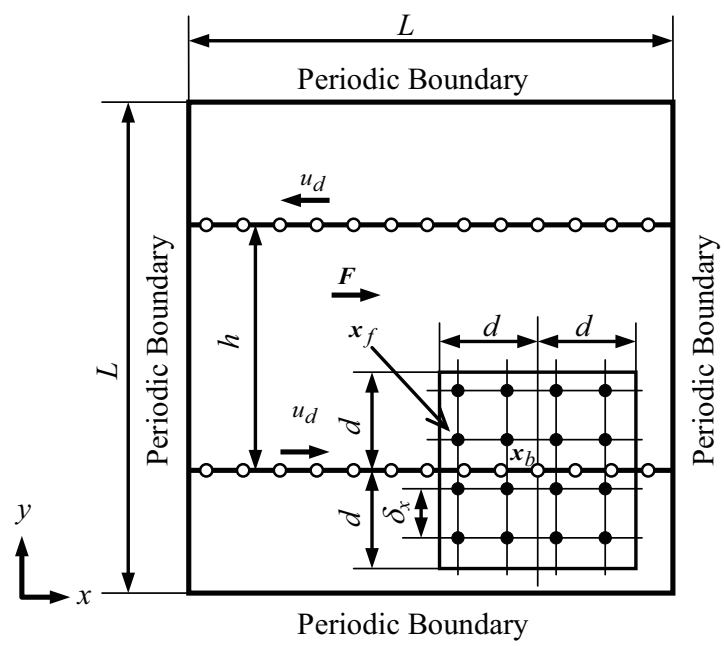

(a) Schematic diagram of a symmetric shear flow and a symmetric Poiseuille flow

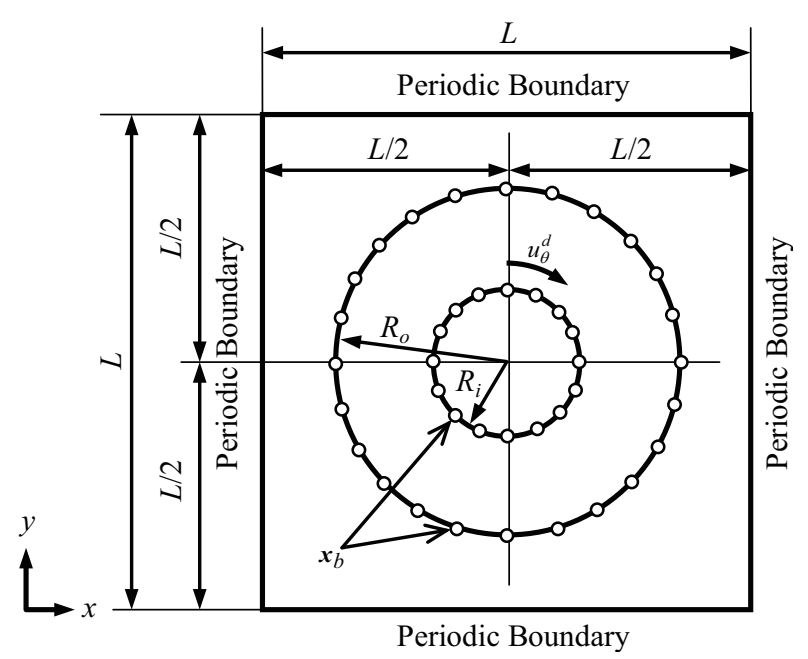

(b) Schematic diagram of a cylindrical Couette flow

FIG. 1. Schematic diagrams of a symmetric shear flow, a symmetric Poiseuille flow, and a cylindrical Couette flow. The circles indicate the boundary nodes $\mathbf{x}_{b}$ and fluid nodes $\mathbf{x}_{f}$ used in the IB-LBM.

Applying the Chapman-Enskog expansion to Eqs. (1) and (4) yields the following macroscopic equations:

$$
\begin{gathered}
\frac{\partial \rho}{\partial t}+\nabla \cdot(\rho \mathbf{u})=0 \\
\frac{\partial \rho \mathbf{u}}{\partial t}+\nabla \cdot(\rho \mathbf{u u})=-\nabla p \\
+\nu \nabla \cdot\left[\rho\left(\nabla \mathbf{u}+(\nabla \mathbf{u})^{T}\right)\right]+\rho \mathbf{G},
\end{gathered}
$$

where $\mathbf{G}$ is a body force. The kinematic viscosity $\nu$ is determined as

$$
\nu=\frac{(2 \tau-1) \delta_{x}^{2}}{6 \delta_{t}}
$$

where $\delta_{x}$ is the Eulerian mesh size, which satisfies $\delta_{x}=$ $c \delta_{t}$. 
As shown in Fig. 1, the immersed boundary method sets the Lagrangian points $\mathbf{x}_{b}$ in uniform Cartesian coordinates to represent the boundary of an arbitrarily shaped body in a viscous incompressible fluid. The force density $\mathbf{G}$ is used to calculate the interaction between the boundary node $\mathbf{x}_{b}$ and the Eulerian grid $\mathbf{x}_{f}$. Among the numerous effective methods of computing the force $\mathbf{G}$ in the IB-LBM, we herein consider three typical methods: the direct forcing method [4], the implicit correction method [6], and the split forcing method [14].

\section{B. Direct forcing method with a single relaxation time [4]}

The direct forcing method evaluates the force density $\mathbf{G}\left(\mathbf{x}_{b}, t\right)$ at the Lagrangian points as follows:

$$
\mathbf{G}\left(\mathbf{x}_{b}, t\right)=\frac{\mathbf{u}^{d}\left(\mathbf{x}_{b}, t\right)-\mathbf{u}\left(\mathbf{x}_{b}, t\right)}{\delta_{t}},
$$

where $\mathbf{u}^{d}$ is the desired wall velocity on the boundary nodes. The velocity $\mathbf{u}\left(\mathbf{x}_{b}\right)$ is evaluated through the interpolation of the velocities at the neighboring fluid nodes $\mathbf{x}_{f}$ using a discrete delta function $D(\mathbf{x})$ as follows:

$$
\mathbf{u}\left(\mathbf{x}_{b}, t\right)=\sum_{f} \mathbf{u}\left(\mathbf{x}_{f}, t\right) D\left(\mathbf{x}_{f}-\mathbf{x}_{b}\right) \delta_{x}^{2} .
$$

In a two-dimensional coordinate system $\mathbf{x}=(x, y)$, $D(\mathbf{x})$ is expressed as

$$
D\left(\mathbf{x}_{f}-\mathbf{x}_{b}\right)=\delta\left(x_{f}-x_{b}\right) \delta\left(y_{f}-y_{b}\right),
$$

where

$$
\delta(r)=\left\{\begin{array}{ll}
\frac{1}{2 d}\left(1+\cos \left(\frac{\pi|r|}{d}\right)\right) & |r| \leq d \\
0 & |r|>d
\end{array} .\right.
$$

For two-way fluid-solid coupling, we need to distribute the force density $\mathbf{G}\left(\mathbf{x}_{b}\right)$ to the fluid nodes. We obtain $\mathbf{G}\left(\mathbf{x}_{f}\right)$ in the Eulerian coordinate system by interpolating $\mathbf{G}\left(\mathbf{x}_{b}\right)$ as

$$
\mathbf{G}\left(\mathbf{x}_{f}, t\right)=\sum_{b}^{N} \mathbf{G}\left(\mathbf{x}_{b}, t\right) D\left(\mathbf{x}_{f}-\mathbf{x}_{b}\right) \Delta s,
$$

where $N$ is the total number of Lagrangian points, and $\Delta s$ is the discrete volume (area) for each Lagrangian point. Equation (12) indicates that the body force at the boundary node is distributed to the fluid nodes in the $2 d \times 2 d$ square shown in Fig. 1(a). For a flat plate of length $L$ in the symmetric shear flows and in the symmetric Poiseuille flows, $\Delta s=L \delta_{x} / N$. For the circular cylinder of radius $R$ in cylindrical Couette flows shown in Fig. 1(b), we use $\Delta s=2 \pi R \delta_{x} / N$, following Reference $[16]$.

The forcing term in Eq. (1) can be written as

$$
F_{k}=\frac{3 \rho \omega_{k}}{c^{2}} \mathbf{G} \cdot \mathbf{e}_{k}
$$

The solution procedure of the IB-LBM based on the direct forcing method with the SRT can be summarized as follows:
(1) Solve Eq. (1) to obtain the distribution functions $f_{k}$ and compute the macroscopic variables $\rho$ and $\mathbf{u}$ using Eq. (2).

(2) Obtain the velocities at the Lagrangian points using Eq. (9), and compute the force density using Eq. (8).

(3) Distribute the force density to Eulerian points using Eq. (12).

(4) Compute the forcing term $F_{k}$ using Eq. (13), and substitute $F_{k}$ into Eq. (1).

(5) Compute the equilibrium distribution functions $f_{k}^{e q}$ using Eq. (4).

(6) Return to step (1).

\section{Implicit correction method with the single relaxation time [6]}

$\mathrm{Wu}$ et al. proposed the IB-LBM using the external forcing term proposed by Guo et al., which can take into account the effect of the external force on the momentum and momentum flux as well as the discrete lattice effect [6, 17].

Guo redefined the fluid density, the velocity, and the forcing term in Eq. (1) as follows:

$$
\begin{array}{r}
\rho=\sum_{k} f_{k}, \quad \mathbf{u}=\frac{1}{\rho} \sum_{k} f_{k} \mathbf{e}_{k}+\delta_{t} \frac{\mathbf{G}}{2} \\
F_{k}=\left(1-\frac{1}{2 \tau}\right) \rho \omega_{k}\left[\frac{3 \mathbf{e}_{k} \cdot \mathbf{G}}{c^{2}}\right. \\
\left.+\frac{9\left(\mathbf{u G}: \mathbf{e}_{k} \mathbf{e}_{k}\right)}{c^{4}}-\frac{3 \mathbf{u} \cdot \mathbf{G}}{c^{2}}\right] .
\end{array}
$$

In the implicit correction method, Eq. (14) is solved in order to obtain the velocity correction $\delta \mathbf{u}=\frac{\delta_{t}}{2} \mathbf{G}$ at all boundary points. This scheme can enforce the non-slip boundary condition and reduce the streamline penetration into the solid surface. Equation (14) can be rewritten as

$$
\rho=\sum_{k} f_{k}, \quad \mathbf{u}=\mathbf{u}^{*}+\delta \mathbf{u}
$$

Here, the intermediate velocity $\mathbf{u}^{*}$ is defined as follows:

$$
\mathbf{u}^{*}=\frac{1}{\rho} \sum_{k} e_{k} f_{k}
$$

Substituting the corrections $\delta \mathbf{u}=\frac{\delta_{t}}{2} \mathbf{G}$ into Eq. (12), we obtain

$$
\delta \mathbf{u}\left(\mathbf{x}_{f}, t\right)=\sum_{b}^{N} \delta \mathbf{u}\left(\mathbf{x}_{b}, t\right) D\left(\mathbf{x}_{f}-\mathbf{x}_{b}\right) \Delta s .
$$

The fluid velocity $\mathbf{u}\left(\mathbf{x}_{b}\right)$ should be equal to the wall velocity $\mathbf{u}^{d}\left(\mathbf{x}_{b}\right)$ at the boundary points $\mathbf{x}_{b}$ in order to satisfy the non-slip boundary condition. From Eqs. (9), (16), 
and (18), we obtain the following simultaneous equations for the unknown velocity $\delta \mathbf{u}$ :

$$
\begin{array}{r}
\mathbf{u}^{d}\left(\mathbf{x}_{b}\right)=\sum_{f} \mathbf{u}^{*}\left(\mathbf{x}_{f}\right) D\left(\mathbf{x}_{f}-\mathbf{x}_{b}\right) \delta_{x}^{2} \\
+\sum_{f} \sum_{b^{\prime}} \delta \mathbf{u}\left(\mathbf{x}_{b^{\prime}}\right) D\left(\mathbf{x}_{f}-\mathbf{x}_{b^{\prime}}\right) \Delta s D\left(\mathbf{x}_{f}-\mathbf{x}_{b}\right) \delta_{x}^{2} .
\end{array}
$$

Equation (19) can be expressed in matrix form as follows:

$$
A \mathbf{X}=\mathbf{B}
$$

where

$$
\begin{gathered}
\mathbf{X}=\left(\delta \mathbf{u}^{1}, \delta \mathbf{u}^{2}, \cdots \delta \mathbf{u}^{N}\right)^{T} \\
\mathbf{B}=\left(\Delta \mathbf{u}_{b}^{1}, \Delta \mathbf{u}_{b}^{2}, \cdots \Delta \mathbf{u}_{b}^{N}\right)^{T} \\
\Delta \mathbf{u}_{b}=\mathbf{u}^{d}\left(\mathbf{x}_{b}\right)-\sum_{f} \mathbf{u}^{*}\left(\mathbf{x}_{f}\right) D\left(\mathbf{x}_{f}-\mathbf{x}_{b}\right) \delta_{x}^{2}
\end{gathered}
$$

Here, ${ }^{T}$ denotes the transpose operator.

The elements of matrix $A$ are computed as a function of the distance between the Lagrangian boundary points and their neighboring Eulerian points. We obtain the unknown velocity correction at all Lagrangian points by solving Eq. (19), and can then easily compute the force as follows:

$$
\mathbf{G}=\frac{2 \delta \mathbf{u}}{\delta_{t}}
$$

We solve the simultaneous equations given by Eq. (20) using a direct method through the lower-upper triangular (LU) decomposition. The solution procedure of the implicit correction method for the IB-LBM can be summarized as follows:

(1) Set the initial values and compute the elements of matrix $A$ and inverse matrix $A^{-1}$ for Eq. (20).

(2) Solve Eq. (1) to obtain the distribution functions $f_{k}$ and compute $\rho, \mathbf{u}^{*}$ using Eqs. (16) and (17).

(3) Solve Eq. (20) for the velocity correction $\delta \mathbf{u}$ and obtain $\mathbf{G}$ at all boundary points using Eq. (22).

(4) Use Eq. (12) to spread the force density to the Eulerian points.

(5) Correct the fluid velocity at Eulerian points using Eq. (16).

(6) Compute the forcing term $F_{k}$ using Eq. (15).

(7) Compute the equilibrium distribution function $f_{k}^{e q}$ using Eq. (4).

(8) Return to step (2).

\section{Split forcing method with the multi-relaxation time [14]}

The dynamic equation adapted to the multi-relaxation time collision scheme is described in concise vector form as follows:

$$
\begin{array}{r}
\left|f\left(\mathbf{x}+\mathbf{e} \delta_{t}, t+\delta_{t}\right)\right\rangle=|f(\mathbf{x}, t)\rangle \\
-M^{-1}\left\{S\left[|R(\mathbf{x}, t)\rangle-\left|R^{(e q)}(\mathbf{x}, t)\right\rangle\right]\right. \\
\left.+(I-S / 2) M \delta_{t}|F(\mathbf{x}, t)\rangle\right\},
\end{array}
$$

where $|f\rangle$ is the distribution function in vector form, $M$ is the transformation matrix, $S$ is the diagonal relaxation matrix, $|R\rangle$ is the moment vector, $\left|R^{(e q)}\right\rangle$ is the equilibrium moment vector, and $I$ is the unity matrix.

For the D2Q9 model, the standard multi-relaxation time scheme is organized as follows:

$$
\begin{aligned}
& |f\rangle=\left(f_{0}, f_{1}, f_{2}, f_{3}, f_{4}, f_{5}, f_{6}, f_{7}, f_{8}\right)^{T}, \\
& S=\operatorname{diag}\left(0, s_{e}, s_{\epsilon}, 0, s_{q}, 0, s_{q}, s_{v}, s_{v}\right), \\
& |R\rangle=\left(\rho, e, \epsilon, j_{x}, q_{x}, j_{y}, q_{y}, p_{x x}, p_{x y}\right)^{T} . \\
& \left|R^{(e q)}\right\rangle=\rho\left(1,-2+3\left(u^{2}+v^{2}\right), 1-3\left(u^{2}+v^{2}\right),\right. \\
& \left.u,-u, v,-v, u^{2}-v^{2}, u v\right)^{T}, \\
& M=\left(\begin{array}{rrrrrrrrr}
1 & 1 & 1 & 1 & 1 & 1 & 1 & 1 & 1 \\
-4 & -1 & -1 & -1 & -1 & 2 & 2 & 2 & 2 \\
4 & -2 & -2 & -2 & -2 & 1 & 1 & 1 & 1 \\
0 & 1 & 0 & -1 & 0 & 1 & -1 & -1 & 1 \\
0 & -2 & 0 & 2 & 0 & 1 & -1 & -1 & 1 \\
0 & 0 & 1 & 0 & -1 & 1 & 1 & -1 & -1 \\
0 & 0 & -2 & 0 & 2 & 1 & 1 & -1 & -1 \\
0 & 1 & -1 & 1 & -1 & 0 & 0 & 0 & 0 \\
0 & 0 & 0 & 0 & 0 & 1 & -1 & 1 & -1
\end{array}\right),
\end{aligned}
$$

where $\mathbf{u}=(u, v)$. The matrix $M$ projects $|f\rangle$ onto $|R\rangle$ such that $|R\rangle=M|f\rangle$, and $\left|R^{(e q)}\right\rangle=M\left|f^{(e q)}\right\rangle$.

Here, $|F\rangle$ represents the forcing term proposed by Guo [17].

$$
F_{k}=\rho \omega_{k}\left[\frac{3 \mathbf{e}_{k} \cdot \mathbf{G}}{c^{2}}+\frac{9\left(\mathbf{u G}: \mathbf{e}_{k} \mathbf{e}_{k}\right)}{c^{4}}-\frac{3 \mathbf{u} \cdot \mathbf{G}}{c^{2}}\right] .
$$

The kinematic viscosity of the MRT model is related to the relaxation time parameter $s_{v}$ :

$$
\nu=\frac{\delta_{x}^{2}}{3 \delta_{t}}\left(\frac{1}{s_{v}}-\frac{1}{2}\right) .
$$

The theoretical analysis for the symmetric shear flow yields the following equation:

$$
s_{q}=\frac{8-4 s_{v}}{4+7 s_{v}}
$$

to reduce the boundary slip. Lu proved that no special restrictions need to be imposed on $s_{e}$ or $s_{\epsilon}$ [14]. In Eq. (25), we assume that $s_{e}=s_{v}$ and $s_{\epsilon}=s_{v}$. 
The following direct forcing formula [18] is chosen for the split forcing method:

$$
\mathbf{G}\left(\mathbf{x}_{b}, t\right)=2 \frac{\mathbf{u}^{d}\left(\mathbf{x}_{b}, t+\delta_{t}\right)-\mathbf{u}^{*}\left(\mathbf{x}_{b}, t+\delta_{t}\right)}{\delta_{t}},
$$

where $\mathbf{u}^{*}$ is an intermediate velocity defined by Eq. (17).

The procedure of the split forcing method with the multi-relaxation time can be concisely illustrated as follows:

(1) Solve Eq. (23) without the forcing term as

$$
\begin{array}{r}
\left|f\left(\mathbf{x}+\mathbf{e} \delta_{t}, t+\delta_{t}\right)\right\rangle=|f(\mathbf{x}, t)\rangle \\
-M^{-1} S\left[|R(\mathbf{x}, t)\rangle-\left|R^{(e q)}(\mathbf{x}, t)\right\rangle\right],
\end{array}
$$

and compute $\rho\left(\mathbf{x}_{f}\right)$ and $\mathbf{u}^{*}\left(\mathbf{x}_{f}\right)$ using Eqs. (16) and (17).

(2) Interpolate the velocities $\mathbf{u}^{*}\left(\mathbf{x}_{b}\right)$ from $\mathbf{u}^{*}\left(\mathbf{x}_{f}\right)$ using Eq. (9), and compute $\mathbf{G}\left(\mathbf{x}_{b}\right)$ using Eq. (32).

(3) Compute $\mathbf{G}\left(\mathbf{x}_{f}\right)$ using Eq. (12).

(4) Compute the forcing term $F_{k}$ using Eq. (29), and solve Eq. (23) to obtain the distribution functions $\left|f\left(\mathbf{x}+\mathbf{e} \delta_{t}, t+\delta_{t}\right)\right\rangle$.

(5) Compute the macroscopic variables $\rho$ and $\mathbf{u}$ using Eq. (14), and compute $f_{k}^{e q}$ using Eq. (4).

(6) Return to step (1).

In the following section, we numerically and analytically compare the effects of reducing the boundary slip of the TRT and MRT models. Before presenting the numerical and analytical solutions of the TRT-based IBLBMs, we summarize the algorithms of the direct forcing methods and of the implicit correction methods that use more than one relaxation time.

\section{E. Direct forcing method with multiple relaxation times}

The TRT collision model takes the counterpart of each velocity vector $\mathbf{e}_{\bar{k}}$ that faces the opposite direction of $\mathbf{e}_{k}$, i.e., $\mathbf{e}_{\bar{k}}=-\mathbf{e}_{k}$. For instance, $\mathbf{e}_{\overline{1}}=-\mathbf{e}_{1}=\mathbf{e}_{3}$ in the D2Q9 model. The distribution function $f_{k}$ and its counterpart with the opposite velocity $f_{\bar{k}}$ generate the symmetric (even) $f_{k}^{+}$and antisymmetric (odd) components $f_{k}^{-}[10]:$

$$
\begin{aligned}
& f_{k}^{ \pm}=\frac{f_{k} \pm f_{\bar{k}}}{2}, \quad f_{k}^{ \pm(e q)}=\frac{f_{k}^{(e q)} \pm f_{\bar{k}}^{(e q)}}{2} \\
& f_{k}=f_{k}^{+}+f_{k}^{-}, \quad f_{k}^{(e q)}=f_{k}^{+(e q)}+f_{k}^{-(e q)}
\end{aligned}
$$

For the rest particle, $f_{0}^{+}=f_{0}, f_{0}^{+(e q)}=f_{0}^{(e q)}, f_{0}^{-}=0$, and $f_{0}^{-(e q)}=0$.

The discrete evolution equation solved in the LBM with the TRT collision operator is given by

$$
\begin{gathered}
f_{k}\left(\mathbf{x}+\mathbf{e}_{k} \delta_{t}, t+\delta_{t}\right)=f_{k}(\mathbf{x}, t)-\frac{f_{k}^{+}(\mathbf{x}, t)-f_{k}^{+(e q)}(\mathbf{x}, t)}{\tau_{+}} \\
-\frac{f_{k}^{-}(\mathbf{x}, t)-f_{k}^{-(e q)}(\mathbf{x}, t)}{\tau_{-}}+\delta_{t} F_{k}(\mathbf{x}, t),
\end{gathered}
$$

where $\tau_{ \pm}$are the relaxation times. When $\tau_{+}=\tau_{-}=\tau$, Eq. (35) becomes equivalent to Eq. (1) with the single relaxation time. The kinematic viscosity $\nu$ is determined as

$$
\nu=\frac{\left(2 \tau_{+}-1\right) \delta_{x}^{2}}{6 \delta_{t}}
$$

In order to reduce the boundary velocity slip, we obtain the following equation:

$$
\tau_{-}=\frac{3 \tau_{+}+3}{4 \tau_{+}-2}
$$

A detailed description of this condition is presented in the next section. The solution procedure is carried out as follows:

(1) Compute the $f_{k}^{ \pm}$and $f_{k}^{ \pm e q}$ using Eq. (34).

(2) Compute $f_{k}, \rho\left(\mathbf{x}_{f}\right)$, and $\mathbf{u}\left(\mathbf{x}_{f}\right)$ using Eqs. (2) and (35).

(3) Obtain $\mathbf{u}\left(\mathbf{x}_{b}\right)$ from $\mathbf{u}\left(\mathbf{x}_{f}\right)$ using Eq. (9).

(4) Compute $\mathbf{G}\left(\mathbf{x}_{\mathbf{b}}\right)$ using Eq. (8), and distribute $\mathbf{G}\left(\mathbf{x}_{\mathbf{b}}\right)$ to Eulerian points using Eq. (12).

(5) Compute $F_{k}$ and $f_{k}^{e q}$ using Eqs. (4) and (13).

(6) Return to step (1).

When the multi-relaxation time collision operator is applied to the direct forcing method, the distribution functions evolve according to the following equation:

$$
\begin{gathered}
\left|f\left(\mathbf{x}+\mathbf{e} \delta_{t}, t+\delta_{t}\right)\right\rangle=|f(\mathbf{x}, t)\rangle \\
-M^{-1} S\left[|R(\mathbf{x}, t)\rangle-\left|R^{(e q)}(\mathbf{x}, t)\right\rangle\right]+\delta_{t}|F(\mathbf{x}, t)\rangle
\end{gathered}
$$

where, $S, R$, and $M$ are given by Eqs. (25), (27), and (28), respectively. The relation between $s_{q}$ and $s_{v}$ for the reduction of the boundary velocity slip is given by

$$
s_{q}=\frac{4-2 s_{v}}{3+3 s_{v}} .
$$

\section{F. Implicit correction method with multiple relaxation times}

In order to apply the TRT collision operator to the implicit correction method, we divide the forcing term defined by Eq. (29) into the odd and even parts,

$$
F_{k}^{ \pm}=\frac{F_{k} \pm F_{\bar{k}}}{2}
$$

The kinetic equation of $f_{k}$ in the lattice Boltzmann method with the TRT collision operator uses the extra forcing term proposed by Guo [17] as follows:

$$
\begin{gathered}
f_{k}\left(\mathbf{x}+\mathbf{e}_{k} \delta_{t}, t+\delta_{t}\right)=f_{k}(\mathbf{x}, t) \\
-\frac{f_{k}^{+}(\mathbf{x}, t)-f_{k}^{+(e q)}(\mathbf{x}, t)}{\tau_{+}}+\delta_{t}\left(1-\frac{1}{2 \tau_{+}}\right) F_{k}^{+}(\mathbf{x}, t) \\
-\frac{f_{k}^{-}(\mathbf{x}, t)-f_{k}^{-(e q)}(\mathbf{x}, t)}{\tau_{-}}+\delta_{t}\left(1-\frac{1}{2 \tau_{-}}\right) F_{k}^{-}(\mathbf{x}, t) .
\end{gathered}
$$


In order to reduce the velocity slip, the relaxation time $\tau_{-}$is given by

$$
\tau_{-}=\frac{4 \tau_{+}+7}{8 \tau_{+}-4} .
$$

The solution procedure of the implicit correction method with the TRT (TRT implicit correction method) can be summarized as follows:

(1) Set the initial values, and compute the elements of matrix $A$ and inverse matrix $A^{-1}$ for Eq. (20).

(2) Compute $f_{k}^{ \pm}, f_{k}^{ \pm e q}, F_{k}^{ \pm}$using Eqs. (34) and (40).

(3) Solve Eq. (41) to obtain the distribution functions $f_{k}$, and use Eqs. (16) and (17) to compute the macroscopic variables $\rho, \mathbf{u}^{*}$.

(4) Solve Eq. (20) using the LU decomposition to obtain $\delta \mathbf{u}$.

(5) Obtain $\mathbf{G}$ at Lagrangian points using Eq. (22).

(6) Use Eq. (12) to spread $\mathbf{G}$ to the Eulerian points.

(7) Correct the fluid velocity at Eulerian points using Eq. (16).

(8) Compute $f_{k}^{e q}$ and $F_{k}$ using Eqs. (4) and (29).

(9) Return to step (2).

The lattice Boltzmann method uses the kinematic equation (23) for the implicit correction method using the multi-relaxation time collision scheme. The relaxation time between $s_{q}$ and $s_{v}$ for the MRT implicit correction method for the reduction of the velocity slip is the same as that for the MRT split forcing method, i.e., $s_{q}=\left(8-4 s_{v}\right) /\left(4+7 s_{v}\right)$, as shown in Eq. (31).

\section{RESULTS AND DISCUSSION}

\section{A. Numerical results}

In this section, we numerically investigate the boundary slip of the IB-LBM schemes with the different LBMs and forcing methods described in the previous section.

Figure 2 shows the profiles of the velocity normalized with respect to the characteristic velocity in the half computational domain for the calculations of the symmetric shear flows, the symmetric Poiseuille flows, and the cylindrical Couette flows by the direct forcing method with a single relaxation time (SRT direct forcing method) shown in Section II B. The convergence criterion for all calculations is

$$
\max \frac{\left\|\mathbf{u}\left(t+\delta_{t}\right)|-| \mathbf{u}(t)\right\|}{u_{0}} \leq 10^{-8},
$$

where $u_{0}$ denotes the characteristic velocity.

When the numerical solution does not satisfy Eq. (43) due to numerical oscillation, we use the results at time $t=100,000 \delta_{t}$ as the converged solutions. The computational domain with the periodic boundary is covered by $200 \times 200$ grid points. In the simulation of the symmetric

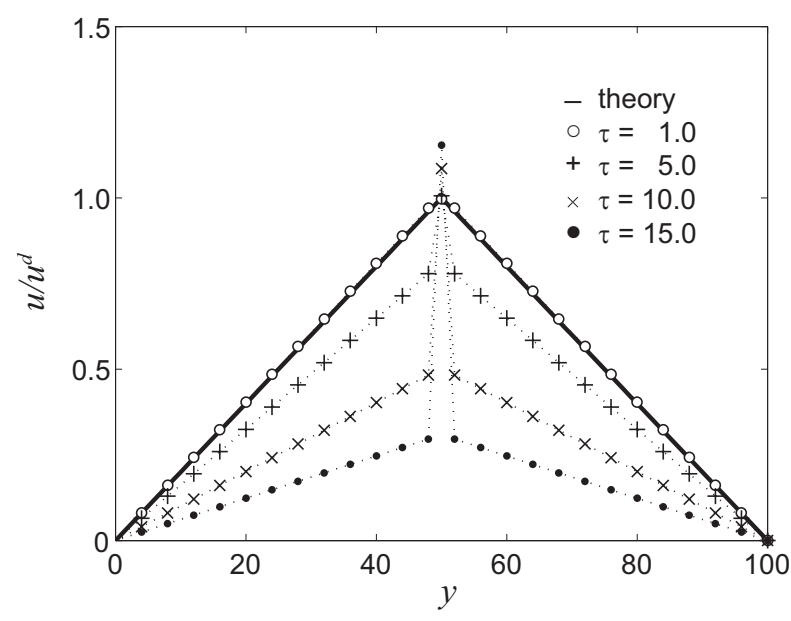

(a) Symmetric shear flow

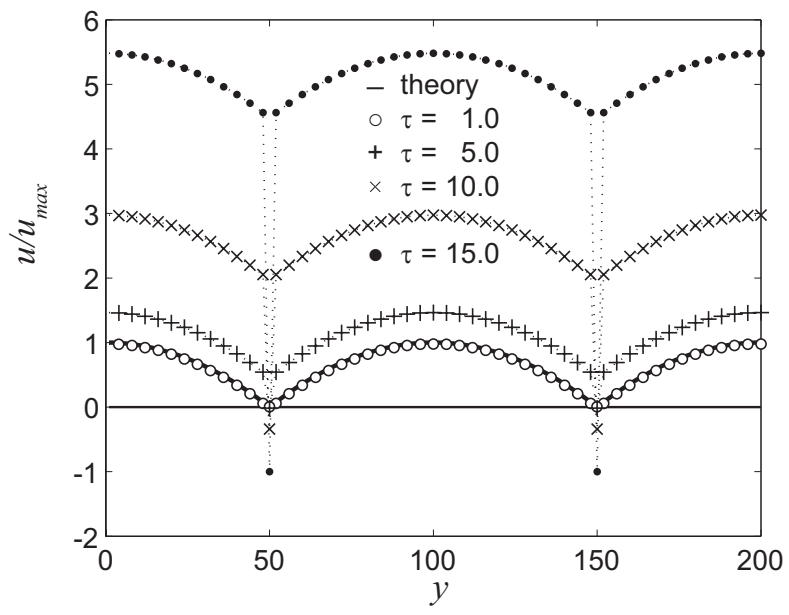

(b) Symmetric Poiseuille flow

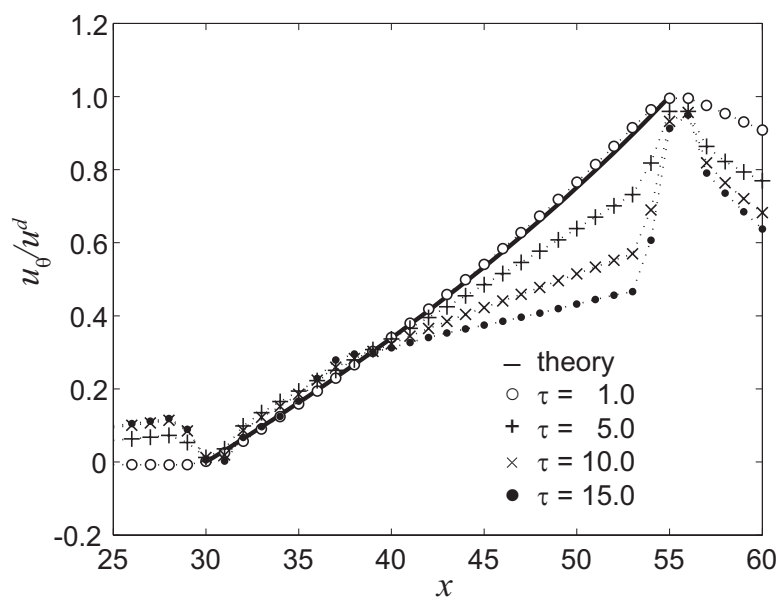

(c) Cylindrical Couette flow

FIG. 2. Velocity profiles calculated by the SRT direct forcing method for the symmetric shear flows, the symmetric Poiseuille flows, and the cylindrical Couette flows normalized with respect to the characteristic velocity. The relaxation parameter $\tau$ is varied as $1,5,10$, and 15 . The thick line indicates the exact velocity profile. 


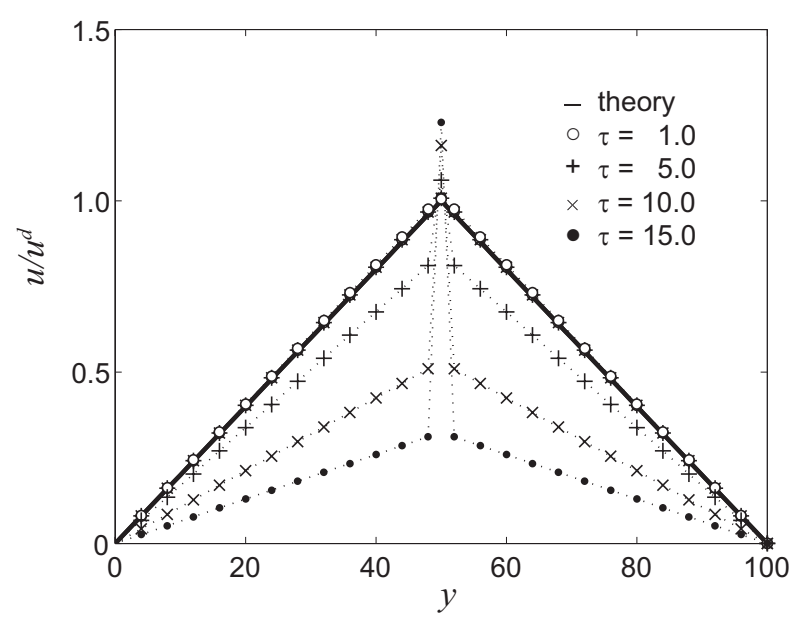

(a) Symmetric shear flow

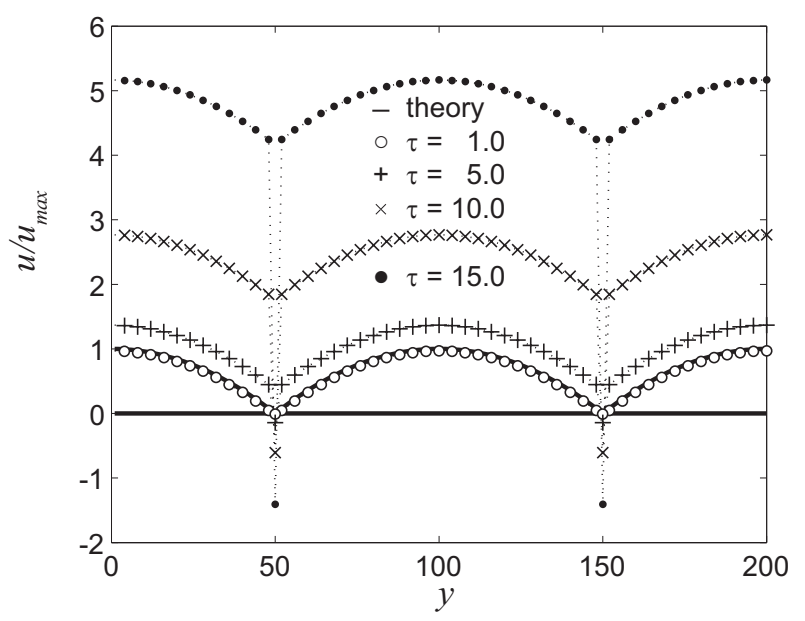

(b) Symmetric Poiseuille flows

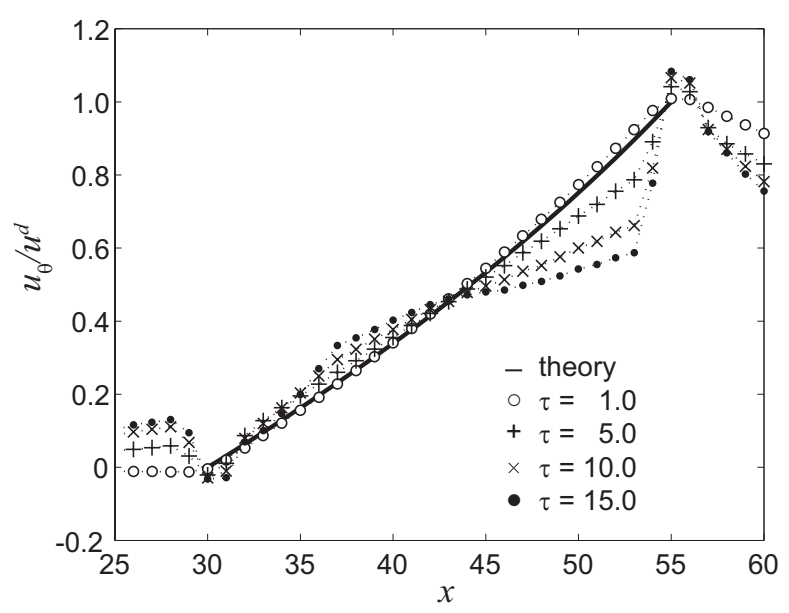

(c) Cylindrical Couette flow

FIG. 3. Velocity profiles calculated by the SRT implicit correction method for the symmetric shear flows, the symmetric Poiseuille flows, and the cylindrical Couette flows normalized with respect to the characteristic velocity. The relaxation parameter $\tau$ is varied as $1,5,10$, and 15 . The thick line indicates the exact velocity profile.

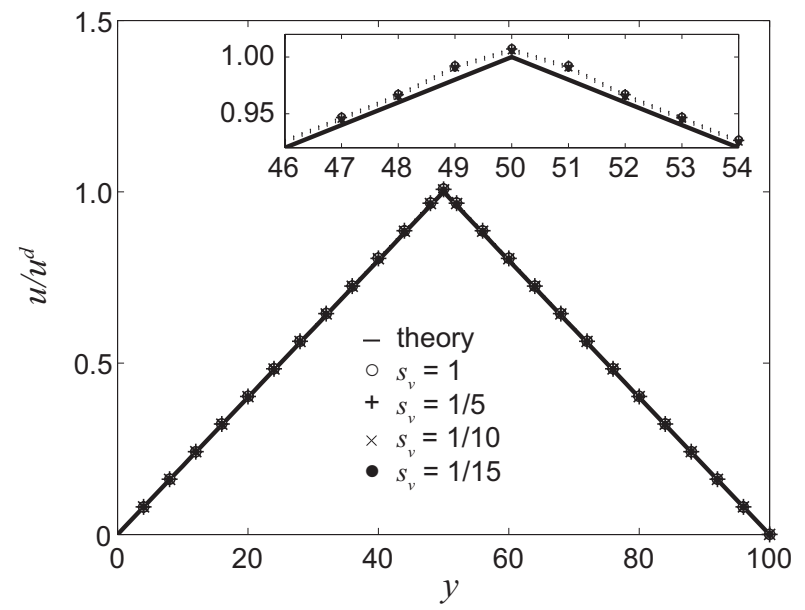

(a) Symmetric shear flow

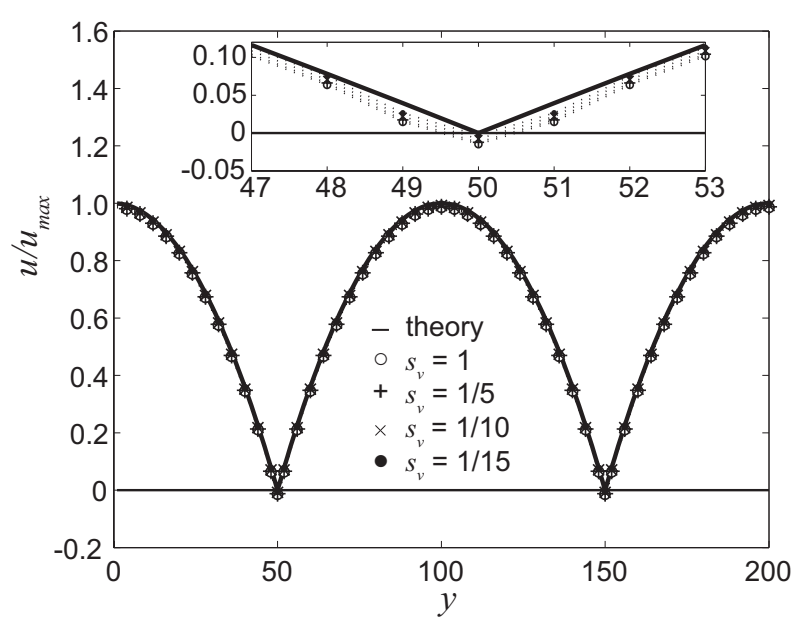

(b) Symmetric Poiseuille flows

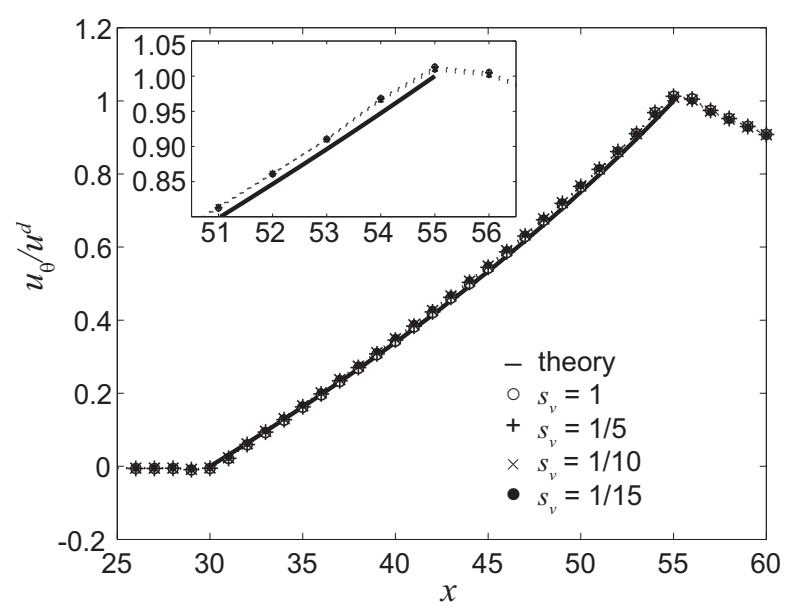

(c) Cylindrical Couette flow

FIG. 4. Velocity profiles calculated using the MRT split forcing method for the symmetric shear flows, the symmetric Poiseuille flows, and the cylindrical Couette flows normalized with respect to the characteristic velocity. The relaxation parameter $s_{v}$ is varied as $1,1 / 5,1 / 10$, and $1 / 15$. The thick line indicates the exact velocity profile. 
shear flows shown in Fig. 1(a), two plates moving at equal and opposite horizontal velocities $u^{d}$ and $-u^{d}$ are placed at $y=50 \delta_{x}$ and $y=150 \delta_{x}$. The velocity $u^{d}=0.01 c$ is set at all Lagrangian points of the boundary, and the distance between the two plates is $h=100$. The body force $F=0$, and $\delta_{x}=\delta_{t}=c=1$ are used.

The symmetric Poiseuille flow problem uses the computational domain shown in Fig. 1(a). A constant body force, $F=10^{-7}$, drives the fluid in the horizontal direction ( $x$-axis). In the simulation of the symmetric Poiseuille flow, two plain surfaces at $y=50 \delta_{x}$ and at $y=150 \delta_{x}$ remain at rest, i.e., $u^{d}=0.0$.

The exact solution for the tangential velocity of the cylindrical Couette flow is given by

$$
\hat{u}_{\theta}(R)=u_{\theta}^{d} \frac{R / R_{o}-R_{o} / R}{R_{i} / R_{o}-R_{o} / R_{i}},
$$

where $R$ is the radial coordinate. The radii of the outer ring, $R_{o}$, and the inner ring, $R_{i}$, are $70 \delta_{x}$ and $45 \delta_{x}$, respectively. The outer ring is at rest, and the velocity of the inner cylinder, $u_{\theta}^{d}$, is 0.01c. As shown in Fig. 1(b), the two rings are placed at the center of the simulation domain and are covered by $200 \delta_{x} \times 200 \delta_{x}$ grid points. As indicated by Le et al. [9], the high relaxation time induces the velocity slip in the numerical solutions calculated by the SRT direct forcing method in Fig. 2.

Figure 3 shows the profiles of the velocity normalized with respect to the characteristic velocity for the test cases shown in Fig. 2 using the implicit correction method with a single relaxation time (SRT implicit correction method) described in Section II C. The SRT implicit correction method makes the boundary velocity agree with the desired velocity and prevents the streamline penetration through the solid body. In Fig. 3, these advantages over the SRT direct forcing method, however, do not contribute to the reduction in the velocity slip at the high relaxation time.

Figure 4 shows the velocity profiles as calculated by the split forcing method with the multi-relaxation time (MRT split forcing method) in the numerical tests shown in Figs. 2 and 3. The MRT split forcing method is explained in Section II D. By satisfying Eq. (31), the MRT split forcing method succeeds in reducing the velocity slip at high relaxation time. Although the MRT split forcing method reduces the velocity slip, the boundary velocity does not completely agree with the desired velocity for each result in Fig. 4. Equation (31) indicates that the multi-relaxation time model requires only two relaxation times $s_{v}$ and $s_{q}$ to reduce the boundary slip. In a previous study [11], we proved that the TRT collision model is also effective in reducing the boundary slip.

Figure 5 shows the velocity profiles calculated by the IB-LBMs, which are shown in Sections II E and II F, for the symmetric shear flows. For both the direct forcing method and the implicit correction method, the TRT collision model provides numerical results similar to those of the MRT collision model. Although the direct forcing method can reduce the velocity slip by using more than one relaxation time, the disagreement between the numerical and theoretical solutions increases with the relaxation time in Figs. 5(a) and 5(c). The implicit correction method reduces both the boundary slip and the disagreement between the numerical and theoretical solutions in Figs. 5(b) and 5(d).

In the following section, in order to verify the effect of the two relaxation times on the reduction of the boundary slip observed in Fig. 5, we analyze the velocity distribution in simple two-dimensional steady incompressible shear flows following the procedure carried out by Le et al. [9] and He et al. [15].

\section{B. An analysis of the IB-LBMs for symmetric shear flows}

In the calculation of the symmetric shear flows, we have

$$
\rho=\text { const }, \quad v=0, \quad \frac{d u}{d x}=0, \quad \text { and } \frac{d u}{d t}=0,
$$

where $\mathbf{u}=(u, v)$ and $\mathbf{G}=(G, 0)$ in two dimensions.

As indicated in Appendix A, Eqs. (41) and (45) give

$$
\begin{aligned}
0= & \nu \frac{u_{j+1}-2 u_{j}+u_{j-1}}{\delta_{x}^{2}}+G_{j} \\
& +\phi\left(G_{j+1}-2 G_{j}+G_{j-1}\right),
\end{aligned}
$$

where

$$
\phi=\frac{1+4 \tau_{+}+4 \tau_{-}-8 \tau_{+} \tau_{-}}{12}
$$

for the TRT implicit correction method. From Eq. (46), we obtain the analytical solutions for the normalized boundary slip velocity $u_{j_{0}}^{s} / u^{d}$, the bulk velocity gradient $d u / d y$, and the normalized boundary velocity $u_{j_{0}} / u^{d}$ for the TRT implicit correction method as follows:

$$
\begin{gathered}
\frac{u_{j_{0}}^{s}}{u^{d}}=\frac{-\frac{7}{24}-\frac{\tau_{+}}{6}-\frac{\tau_{-}}{6}+\frac{\tau_{+} \tau_{-}}{3}}{\frac{h}{4}-\frac{13}{32}-\frac{\tau_{+}}{8}-\frac{\tau_{-}}{8}+\frac{\tau_{+} \tau_{-}}{4}}, \\
\frac{\left(\frac{d u}{d y}\right)_{b u l k}}{\frac{2 u^{d}}{h \delta_{x}}}=\frac{\frac{h}{4}}{\frac{h}{4}-\frac{13}{32}-\frac{\tau_{+}}{8}-\frac{\tau_{-}}{8}+\frac{\tau_{+} \tau_{-}}{4}}, \\
\frac{u_{j_{0}}}{u^{d}}=\frac{\frac{h}{4}-\frac{7}{24}-\frac{\tau_{+}}{6}-\frac{\tau_{-}}{6}+\frac{\tau_{+} \tau_{-}}{3}}{\frac{h}{4}-\frac{13}{32}-\frac{\tau_{+}}{8}-\frac{\tau_{-}}{8}+\frac{\tau_{+} \tau_{-}}{4}} .
\end{gathered}
$$

Substituting $\tau_{+}=\tau_{-}$into Eqs. (48) through (50) gives the analytical solution for the SRT implicit correction method.

The distribution function $f_{k}$ in the MRT implicit correction method evolves following the kinematic equation (23), which is also used in the MRT split forcing method. Lu [14] has proved that Eq. (23) gives

$$
\begin{array}{r}
0=\nu \frac{u_{j+1}-2 u_{j}+u_{j-1}}{\delta_{x}^{2}}+G_{j} \\
+\frac{s_{v} s_{q}+4 s_{v}+4 s_{q}-8}{12 s_{v} s_{q}}\left(G_{j+1}-2 G_{j}+G_{j-1}\right) .
\end{array}
$$




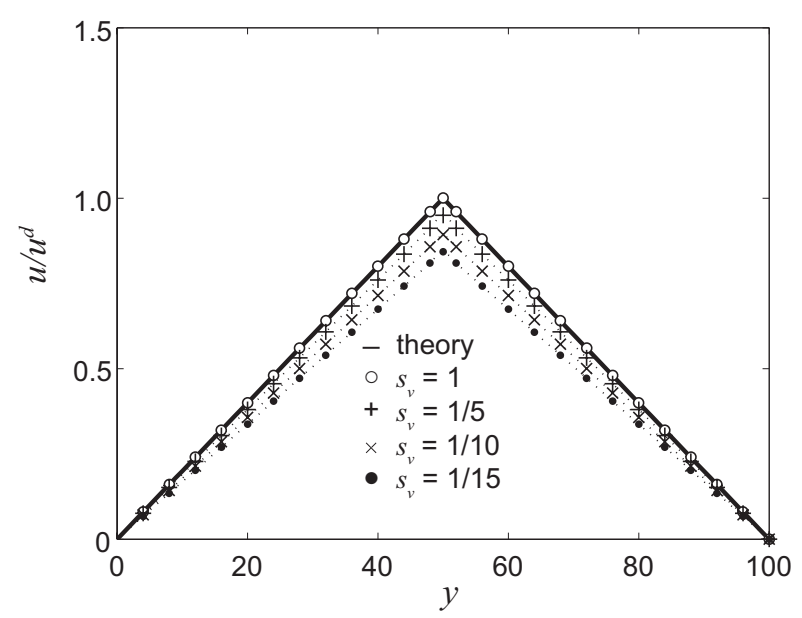

(a) MRT direct forcing method

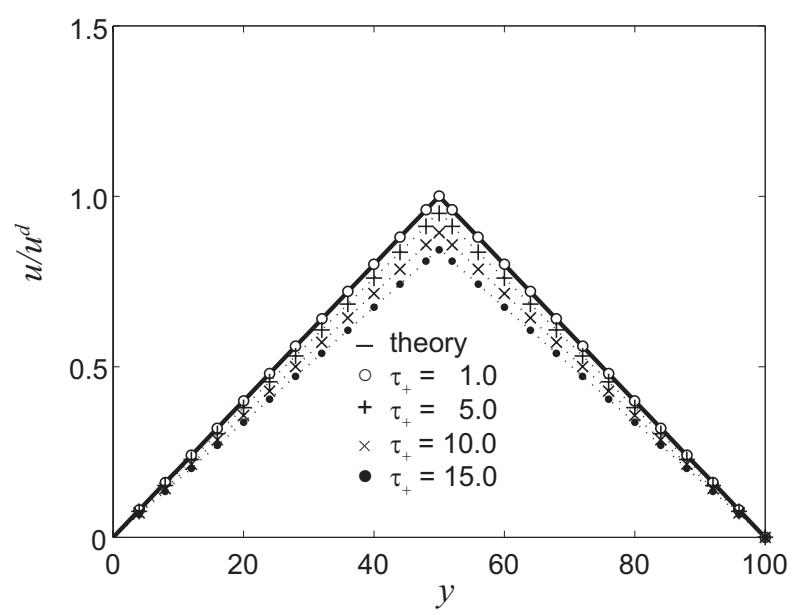

(c) TRT direct forcing method

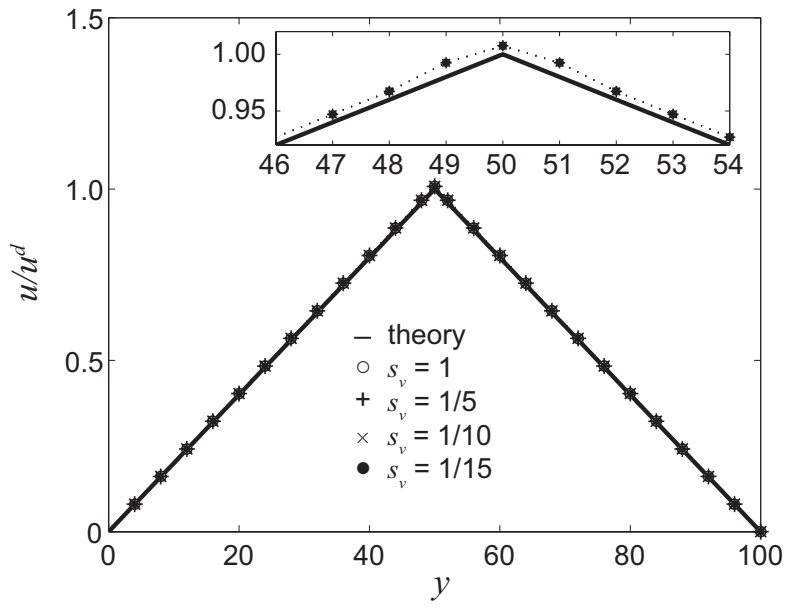

(b) MRT implicit correction method

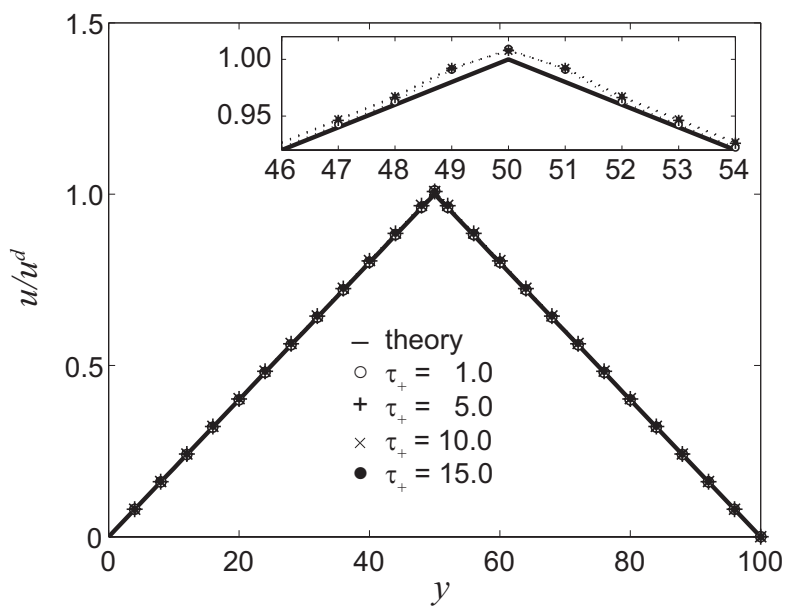

(d) TRT implicit correction method

FIG. 5. Velocity profiles obtained through calculation of the symmetric shear flows. The relaxation parameter $\tau_{+}$is varied as $1,5,10$, and $15\left(s_{v}=1 / \tau_{+}\right)$. The thick line indicates the exact velocity profile.

Since Eq. (46) can be made equivalent to Eq. (51) by replacement of $\tau_{+}$with $1 / s_{v}$ and of $\tau_{-}$with $1 / s_{q}$, the substitution of $\tau_{+}=1 / s_{v}$ and $\tau_{-}=1 / s_{q}$ into Eqs. (48) through (50) gives the solutions for the MRT implicit correction method as follows:

$$
\begin{gathered}
\frac{u_{j_{0}}^{s}}{u^{d}}=\frac{-\frac{7}{24}-\frac{1}{6 s_{v}}-\frac{1}{6 s_{q}}+\frac{1}{3 s_{v} s_{q}}}{\frac{h}{4}-\frac{13}{32}-\frac{1}{8 s_{v}}-\frac{1}{8 s_{q}}+\frac{1}{4 s_{v} s_{q}}}, \\
\frac{\left(\frac{d u}{d y}\right)_{b u l k}}{\frac{2 u^{d}}{h \delta_{x}}}=\frac{\frac{h}{4}}{\frac{h}{4}-\frac{13}{32}-\frac{1}{8 s_{v}}-\frac{1}{8 s_{q}}+\frac{1}{4 s_{v} s_{q}}}, \\
\frac{u_{j_{0}}}{u^{d}}=\frac{\frac{h}{4}-\frac{7}{24}-\frac{1}{6 s_{v}}-\frac{1}{6 s_{q}}+\frac{1}{3 s_{v} s_{q}}}{\frac{h}{4}-\frac{13}{32}-\frac{1}{8 s_{v}}-\frac{1}{8 s_{q}}+\frac{1}{4 s_{v} s_{q}}}
\end{gathered}
$$

Using a similar procedure, Eq. (35) gives the parameter $\phi$ in Eq. (46) for the TRT direct forcing method.

$$
\phi=\frac{3 \tau_{+}+2 \tau_{-}-4 \tau_{+} \tau_{-}}{6} .
$$

Equation (55) gives the analytical solution for the TRT direct forcing method. (See Appendix A for the detailed derivation.)

$$
\begin{gathered}
\frac{u_{j_{0}}^{s}}{u^{d}}=\frac{-\frac{1}{4}-\frac{\tau_{+}}{4}-\frac{\tau_{-}}{6}+\frac{\tau_{+} \tau_{-}}{3}}{\frac{h}{4}-\frac{13}{24}+\frac{7 \tau_{+}}{48}-\frac{\tau_{-}}{8}+\frac{\tau_{+} \tau_{-}}{4}}, \\
\frac{\left(\frac{d u}{d y}\right)_{b u l k}}{\frac{2 u^{d}}{h \delta_{x}}}=\frac{\frac{h}{4}}{\frac{h}{4}-\frac{13}{24}+\frac{7 \tau_{+}}{48}-\frac{\tau_{-}}{8}+\frac{\tau_{+} \tau_{-}}{4}}, \\
\frac{u_{j_{0}}}{u^{d}}=\frac{\frac{h}{4}-\frac{1}{4}-\frac{\tau_{+}}{4}-\frac{\tau_{-}}{6}+\frac{\tau_{+} \tau_{-}}{3}}{\frac{h}{4}-\frac{13}{24}+\frac{7 \tau_{+}}{48}-\frac{\tau_{-}}{8}+\frac{\tau_{+} \tau_{-}}{4}} .
\end{gathered}
$$

The TRT direct forcing method should satisfy Eq. (37) in order to make $u_{j_{0}}^{s}$ equal to zero in Eq. (56). When $\tau_{+}$ and $\tau_{-}$are replaced by $\tau$ in Eqs. (56) through (58), we obtain the analytical solutions for the SRT direct forcing method. 


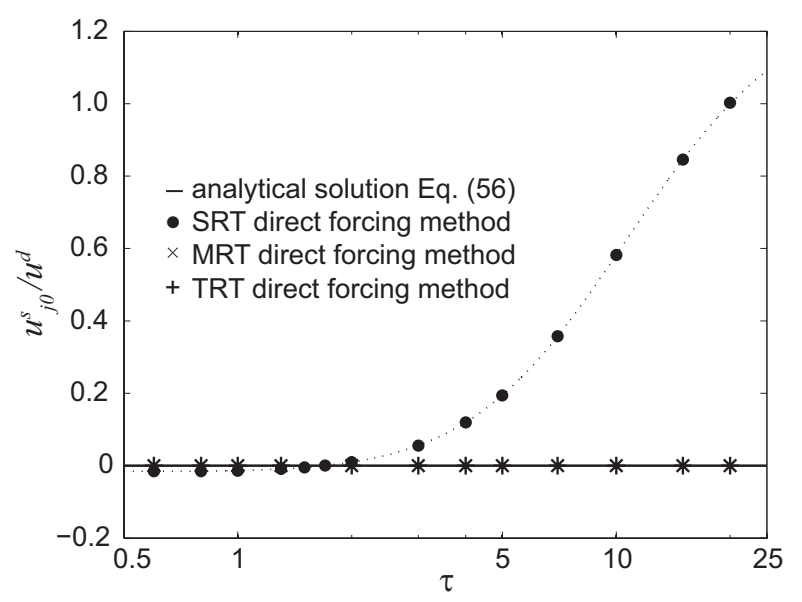

(a-1) Direct forcing method

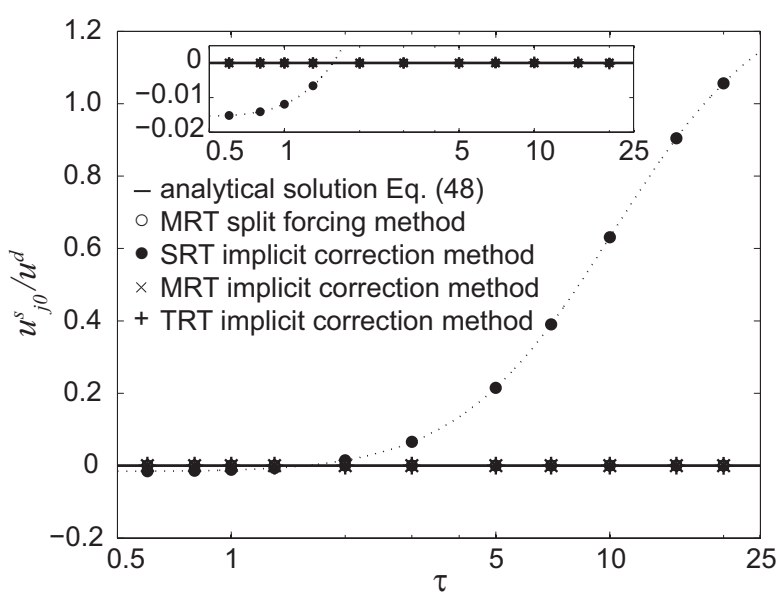

(a-2) Implicit correction method

(a) Boundary slip velocity

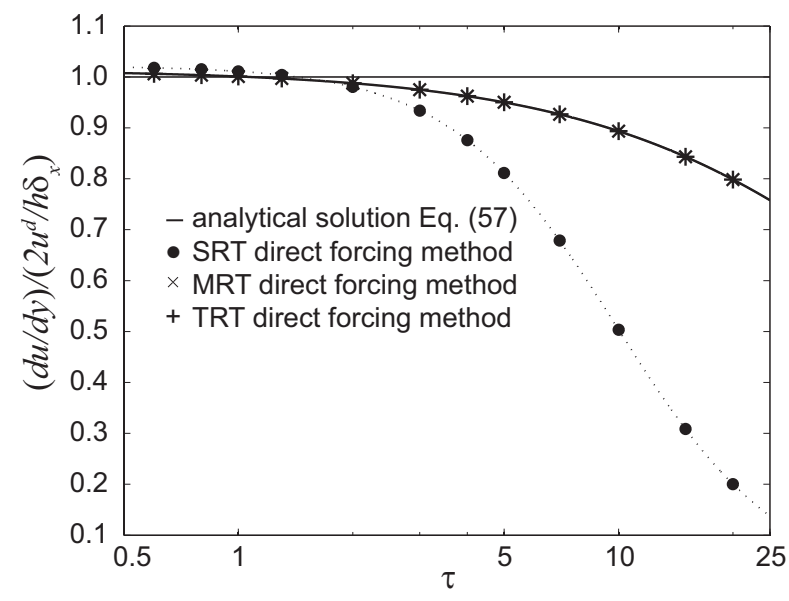

(b-1) Direct forcing method

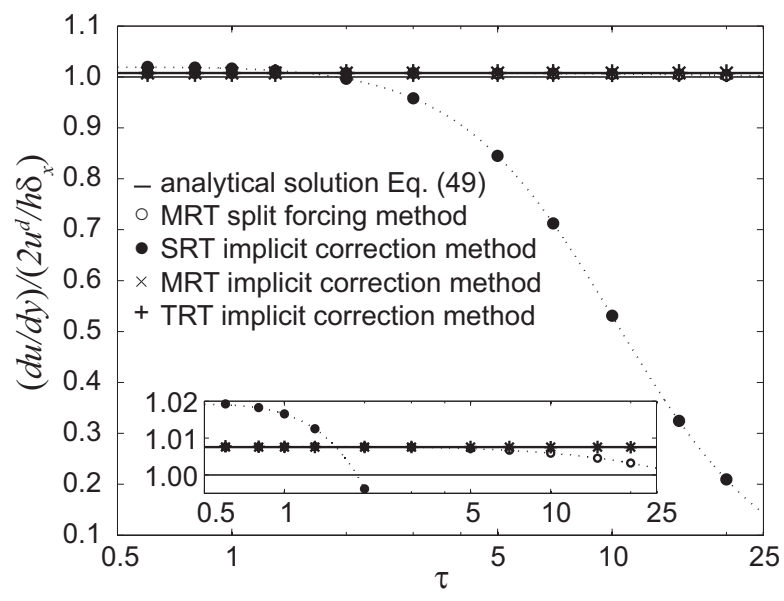

(b-2) Implicit correction method

(b) Velocity gradient

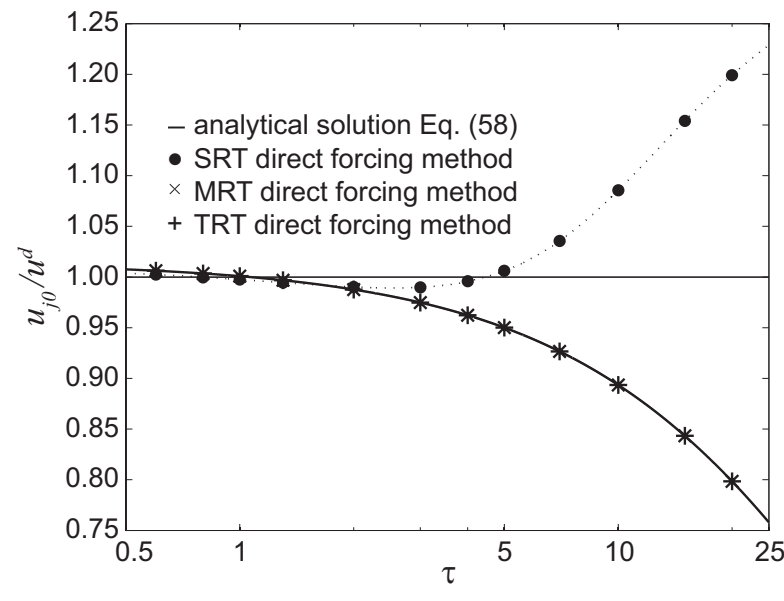

(c-1) Direct forcing method

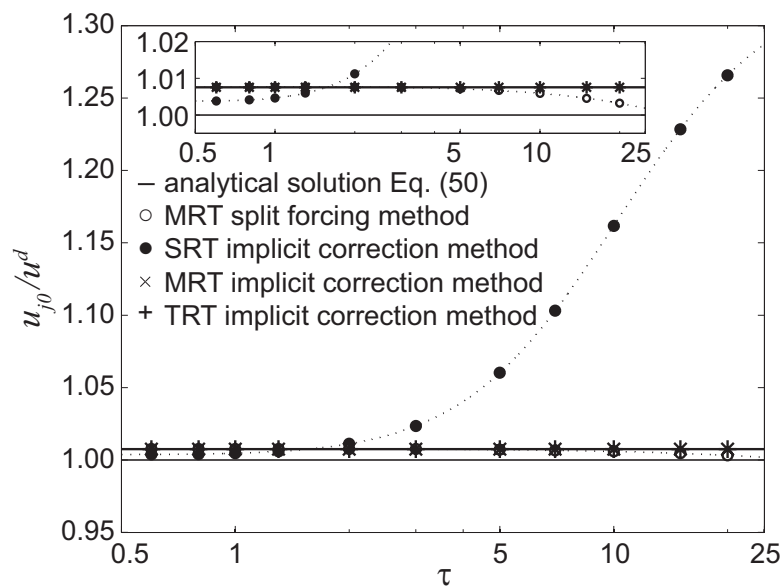

(c-2) Implicit correction method

(c) Fluid velocity at boundary

FIG. 6. Comparison of the analytical and numerical solutions for the direct forcing method and the implicit correction method depending on the relaxation time. The horizontal axis indicates the relaxation time $\tau$, including $\tau_{+}$and $1 / s_{v}$.

For the MRT direct forcing method, we obtain $\phi$ in
Eq. (46) as follows (see Appendix A):

$$
\phi=\frac{3 s_{q}+2 s_{v}-4}{6 s_{v} s_{q}} .
$$


Similarly to the implicit correction method, when $\tau_{+}=$ $1 / s_{v}$ and $\tau_{-}=1 / s_{q}$, Eq. (59) is equivalent to Eq. (55). Equation (59) yields the following analytical solution:

$$
\begin{gathered}
\frac{u_{j_{0}}^{s}}{u^{d}}=\frac{-\frac{1}{4}-\frac{1}{4 s_{v}}-\frac{1}{6 s_{q}}+\frac{1}{3 s_{v} s_{q}}}{\frac{h}{4}-\frac{13}{24}+\frac{7}{48 s_{v}}-\frac{1}{8 s_{q}}+\frac{1}{4 s_{v} s_{q}}}, \\
\frac{\left(\frac{d u}{d y}\right)_{b u l k}}{\frac{2 u^{d}}{h \delta_{x}}}=\frac{\frac{h}{4}}{\frac{h}{4}-\frac{13}{24}+\frac{7}{48 s_{v}}-\frac{1}{8 s_{q}}+\frac{1}{4 s_{v} s_{q}}}, \\
\frac{u_{j_{0}}}{u^{d}}=\frac{\frac{h}{4}-\frac{1}{4}-\frac{1}{4 s_{v}}-\frac{1}{6 s_{q}}+\frac{1}{3 s_{v} s_{q}}}{\frac{h}{4}-\frac{13}{24}+\frac{7}{48 s_{v}}-\frac{1}{8 s_{q}}+\frac{1}{4 s_{v} s_{q}}} .
\end{gathered}
$$

Figure 6 shows the numerical results computed by the IB-LBMs and the analytical solutions. We compare the present solutions to those of the MRT split forcing method. Lu et al. [14] reported the analytical solutions for the MRT split forcing method as follows:

$$
\begin{gathered}
\frac{u_{j_{0}}^{s}}{u^{d}}=\frac{0}{\frac{h}{4}+\frac{8-242 s_{v}-367 s_{v}^{2}}{288 s_{v}\left(4+7 s_{v}\right)}}, \\
\frac{\left(\frac{d u}{d y}\right)_{b u l k}}{\frac{2 u^{d}}{h \delta_{x}}}=\frac{\frac{h}{4}}{\frac{h}{4}+\frac{8-242 s_{v}-367 s_{v}^{2}}{288 s_{v}\left(4+7 s_{v}\right)}} \\
\frac{u_{j_{0}}}{u^{d}}=\frac{\frac{h}{4}}{\frac{h}{4}+\frac{8-242 s_{v}-367 s_{v}^{2}}{288 s_{v}\left(4+7 s_{v}\right)}} .
\end{gathered}
$$

In Fig. 6, the horizontal axis indicates the relaxation time $\tau$ including $\tau_{+}$and $1 / s_{v}$. As predicted from the analytical solutions, Eqs. (48), (52), (56), and (60), the boundary slip disappears for any value of the relaxation times $\tau_{+}$and $s_{v}$ by using the MRT and the TRT in Fig. 6(a). The velocity gradient $(d u / d y)_{b u l k}$ at the boundary node is calculated using the body force $\mathbf{G}$ and

$$
\left(\frac{d u}{d y}\right)_{b u l k}=\frac{G_{0} \delta_{x}}{2 \nu} .
$$

The good agreement for the velocity gradient between the numerical and analytical solutions in Figs. 6(b) verifies the validity of the analytical solutions given by Eqs. (49) and (57). The disagreement between the numerical result $u_{j_{0}}$ and the desired boundary velocity $u^{d}$ in the implicit correction method is less than that for the direct forcing method, as shown in Fig. 6(c). The implicit correction method reveals disagreement between $u_{j_{0}}$ and $u^{d}$ similar to that of the MRT split forcing method in Fig. 6(c-2). We demonstrate analytically and numerically that the TRT collision model has exactly the same effect as the MRT in reducing the boundary slip.

\section{An analysis of the IB-LBMs for symmetric Poiseuille flows}

Figure 7 shows the velocity profiles for the symmetric Poiseuille flow calculated by the direct forcing method and the implicit correction method using multiple relaxation times. As is the case for the symmetric shear flow shown in Fig. 5, the velocity profiles for the TRT model are the same as those for the MRT model obtained by the direct forcing method and by the implicit correction method. Following a procedure similar to that used in the calculation of the symmetric shear flow, we obtain analytical solutions for the symmetric Poiseuille flow driven by a force $F$ along the $x$-axis in Fig. 1(a).

The procedure shown in Appendix B gives the numerical velocity slip:

$$
u_{j_{0}}^{s}=u_{j_{0}}-\bar{u}_{j_{0}}=\frac{F h \delta_{x}^{2}(1+2 \phi)}{4 \nu} .
$$

Substituting Eq. (42) into Eq. (47) gives

$$
\phi=-\frac{1}{2} \text {. }
$$

Equations (31), (37), and (39) can also be used to obtain Eq. (68). Since Eq. (68) makes $u_{j 0}^{s}=0$ in Eq. (67), the IB-LBMs can use the same condition for $\tau_{-}$and for $s_{q}$ to reduce the velocity slip in the symmetric Poiseuille flows as in the symmetric shear flows.

Since $u^{d}=u_{b}$ in the implicit correction method, the boundary error $u_{j_{0}}-u^{d}$ is given as follows (see Appendix B):

$$
u_{j_{0}}-u^{d}=\frac{\delta_{x}^{2} F}{\nu}\left(\frac{h(\phi-1)}{8}+\frac{1}{4}\right),
$$

for the implicit correction method, and

$$
\begin{aligned}
& u_{j_{0}}-u^{d}=u_{j_{0}}-\left(u_{b}+\delta_{t} G_{0}\right) \\
= & \frac{\delta_{x}^{2} F}{\nu}\left(\frac{h(\phi-1)}{8}+\frac{1}{4}+\frac{h \nu \delta_{t}}{\delta_{x}^{2}}\right),
\end{aligned}
$$

for the direct forcing method.

For the symmetric Poiseuille flow, the maximum velocity is given by $u_{\max }=\left(\delta_{x}^{2} h^{2} F\right) /(8 \nu)$ at $j=j_{0}+\frac{h}{2}$. We obtain the velocity differences between $u_{j_{0}}$ and $u^{d}$ normalized with respect to the maximum velocity as follows:

$$
\frac{u_{j_{0}}-u^{d}}{u_{\max }}=\frac{1}{h}\left(\frac{2}{h}-\frac{3}{2}\right),
$$

for the implicit correction method, and

$$
\frac{u_{j_{0}}-u^{d}}{u_{\max }}=\frac{1}{h}\left(\frac{2}{h}-\frac{3}{2}+\frac{8 \nu \delta_{t}}{\delta_{x}^{2}}\right),
$$

for the direct forcing method.

Equations (71) and (72) show that neither the MRT nor the TRT eliminate the boundary error $u_{j_{0}}-u^{d}$. Equation (71) shows that the boundary error of the implicit correction method depends only on the characteristic length $h$. On the other hand, the boundary error of 


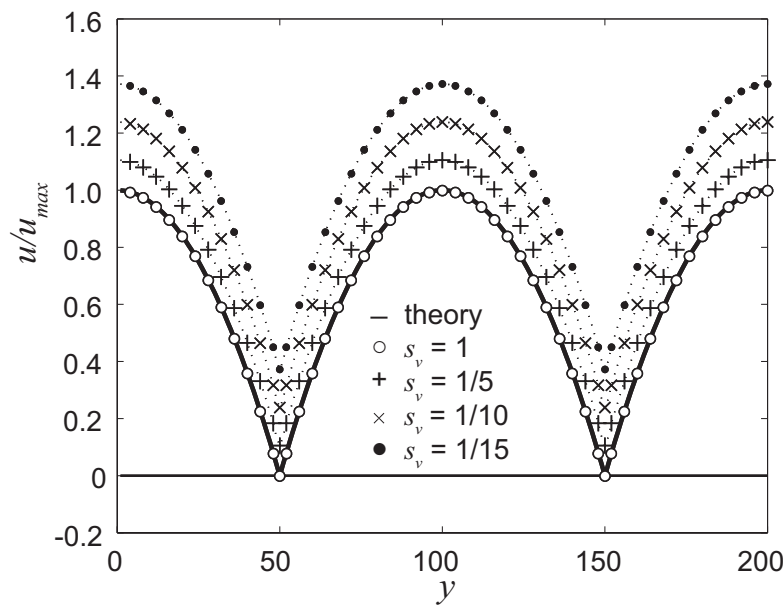

(a) MRT direct forcing method

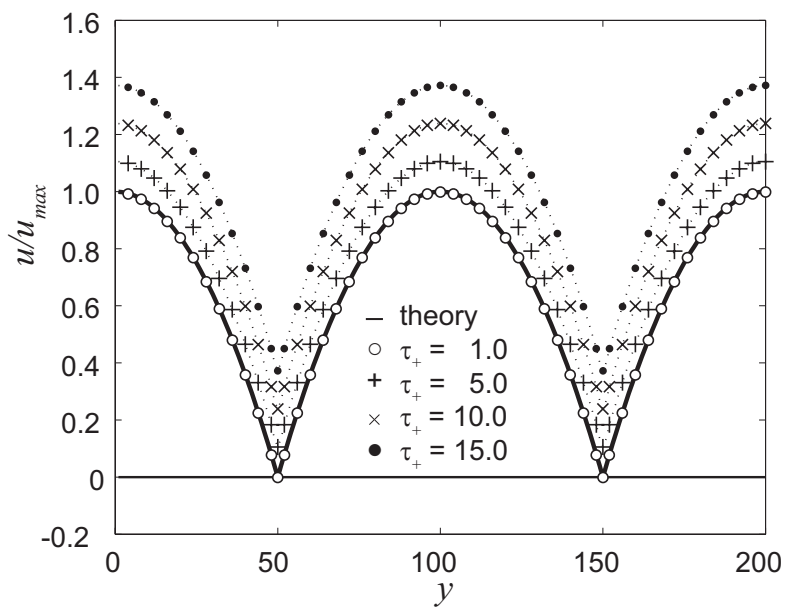

(c) TRT direct forcing method

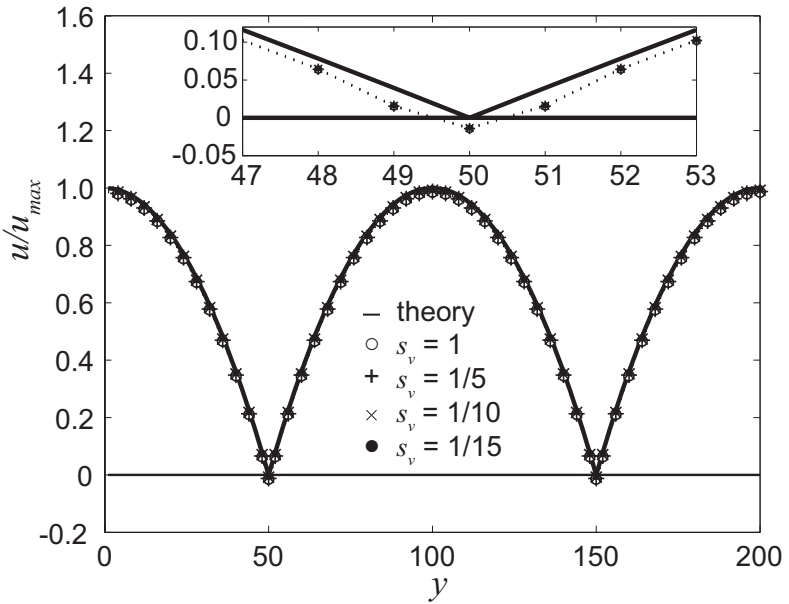

(b) MRT implicit correction method

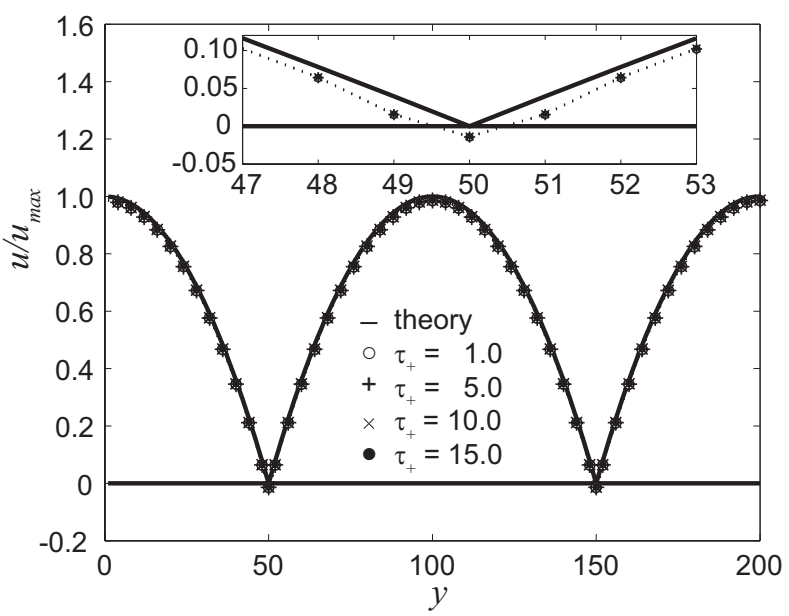

(d) TRT implicit correction method

FIG. 7. Velocity profiles in the calculations of the symmetric Poiseuille flows. The relaxation parameter $\tau_{+}$is varied as $1,2,5$, 10 , and $15\left(s_{v}=1 / \tau_{+}\right)$. The thick line indicates the exact velocity profile.

the direct forcing method increases with the kinematic viscosity $\nu$ in Eq. (72). For the calculation of the symmetric shear flow, Eqs. (42) and (50) give

$$
\frac{u_{j_{0}}}{u^{d}}=\frac{1}{1-\frac{3}{4 h}},
$$

for the implicit correction method. Equations (37) and (58) yield

$$
\frac{u_{j_{0}}}{u^{d}}=\frac{1}{1-\frac{3}{4 h}+\frac{4 \delta_{t} \nu}{h \delta_{x}^{2}}},
$$

for the direct forcing method. Similarly to the symmetric Poiseuille flows, Eq. (73) reveals that the relaxation time does not affect the boundary velocity $u_{j_{0}} / u^{d}$ in the calculation of the symmetric shear flow using the implicit correction method. As shown in Eq. (74), for the direct forcing method, $u_{j_{0}} / u^{d}$ also depends on the kinematic viscosity, which is a function of the relaxation time.
When $\tau_{+}=\tau_{-}=\tau$, Eqs. (67), (69), and (70) give the analytical solutions for the single relaxation time model. For the SRT implicit correction method, Eq. (47) gives

$$
\phi=\frac{1+8 \tau-8 \tau^{2}}{12} .
$$

Substituting Eq. (75) into Eqs. (67) and (69), we obtain

$$
u_{j_{0}}^{s}=u_{j_{0}}-\bar{u}_{j_{0}}=\frac{F h \delta_{x}^{2}\left(7+8 \tau-8 \tau^{2}\right)}{24 \nu},
$$

and

$$
u_{j_{0}}-u^{d}=\frac{\delta_{x}^{2} F\left(24-h\left(11-8 \tau+8 \tau^{2}\right)\right)}{96 \nu} .
$$

For the SRT direct forcing method, Eqs. (55), (67), and (70) give

$$
\phi=\frac{5 \tau-4 \tau^{2}}{6},
$$




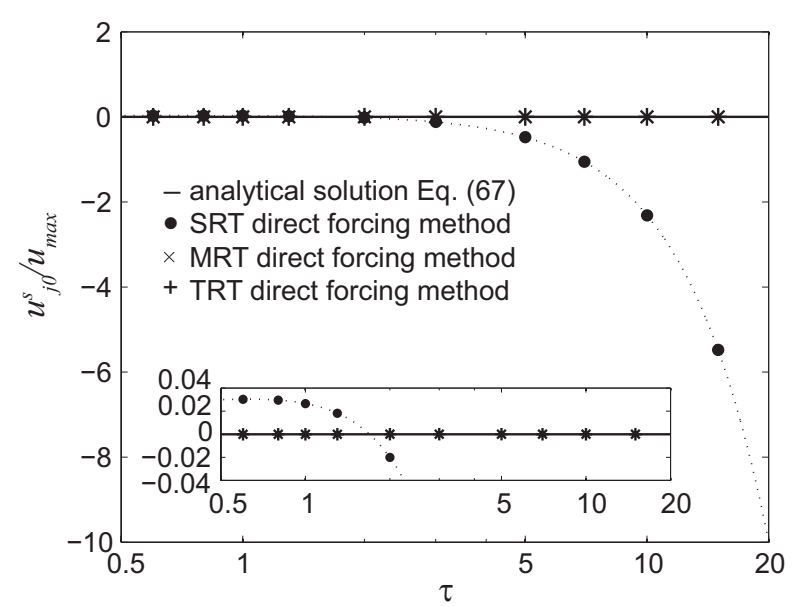

(a-1) Direct forcing method

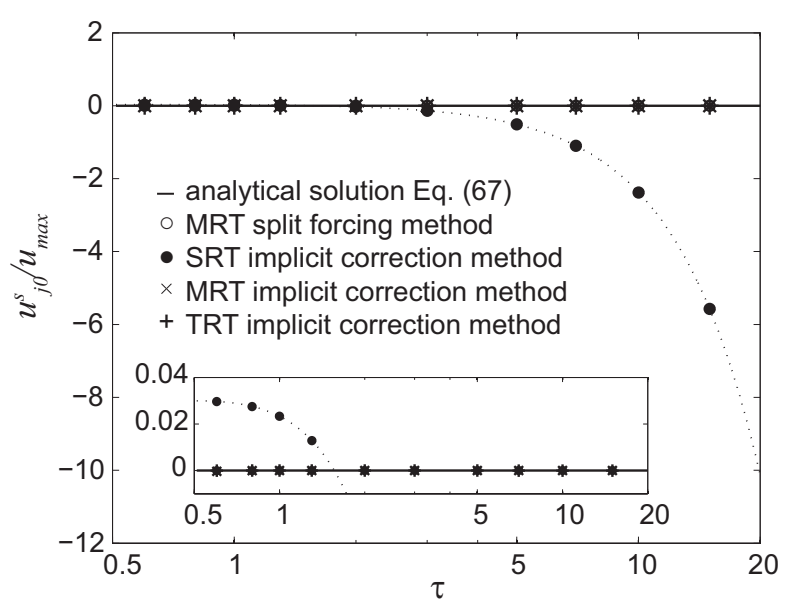

(a-2) Implicit correction method

(a) Boundary slip velocity

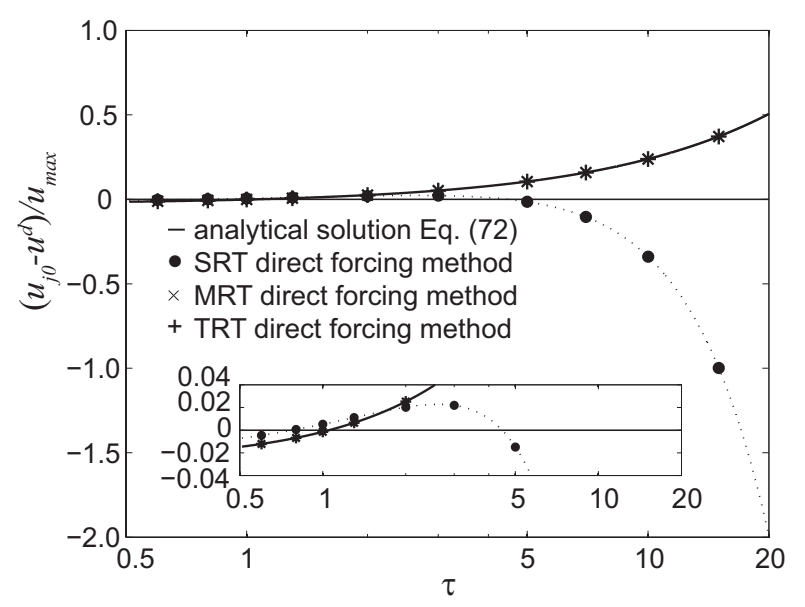

(b-1) Direct forcing method

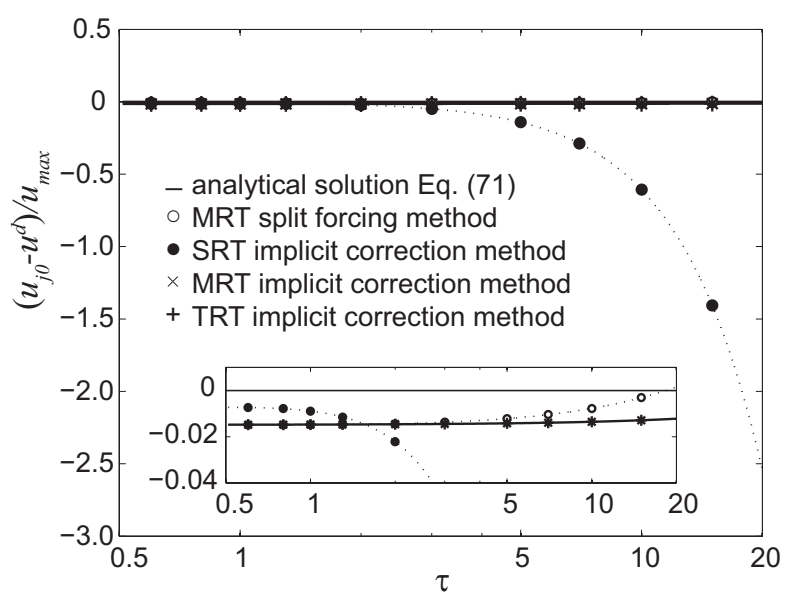

(b-2) Implicit correction method

(b) Fluid velocity at boundary

FIG. 8. Comparison of the analytical and numerical solutions for the direct forcing method and the implicit correction method in the calculation of the symmetric Poiseuille flows. The horizontal axis indicates the relaxation time $\tau$, including $\tau_{+}$and $1 / s_{v}$.

$$
u_{j_{0}}^{s}=u_{j_{0}}-\bar{u}_{j_{0}}=\frac{F h \delta_{x}^{2}\left(3+5 \tau-4 \tau^{2}\right)}{12 \nu},
$$

and

$$
u_{j_{0}}-u^{d}=\frac{\delta_{x}^{2} F\left(12-h\left(14-21 \tau+4 \tau^{2}\right)\right)}{48 \nu} .
$$

Lu et al. obtained the following expression for the split forcing method based on the multi-relaxation time [14]:

$$
\begin{aligned}
u_{j}^{*}= & \frac{1+s_{v}}{3} u_{j}+\frac{2-s_{v}}{6}\left(u_{j+1}+u_{j-1}\right)-\frac{3-2 s_{v}}{2} \delta_{t} G_{j} \\
& -\frac{8+17 s_{v}+21 s_{v}^{2}}{6\left(4+7 s_{v}\right)} \delta_{t}\left(G_{j+1}-2 G_{j}+G_{j-1}\right),
\end{aligned}
$$

where $u_{j}^{*}$ is the intermediate velocity defined by Eq. (17).

Using similar algebraic manipulation, Eq. (81) gives the boundary velocity error as follows:

$$
u_{j_{0}}-u^{d}=\frac{F}{\nu}\left(\frac{1}{2 s_{v}}-\frac{h\left(367 s_{v} s_{v}+242 s_{v}-8\right)}{288 s_{v}\left(4+7 s_{v}\right)}\right) .
$$

Figure 8 shows the numerical and analytical solutions versus the relaxation time. Figure 8(a) shows that the MRT and TRT models succeed in reducing the boundary slip. As predicted by Eq. (72), the direct forcing method increases the difference between the velocity $u_{j_{0}}$ and the desired velocity $u^{d}$ with the relaxation time in Fig. 8(b-1). Figure 8(b-2) shows the small constant difference between $u_{j_{0}}$ and $u^{d}$ in the solutions of the implicit correction method using the TRT or the MRT. The results shown in Fig. 8 demonstrate both analytically and numerically that the TRT contributes to the reduction of the boundary slip for symmetric Poiseuille flows as effectively as the MRT. 


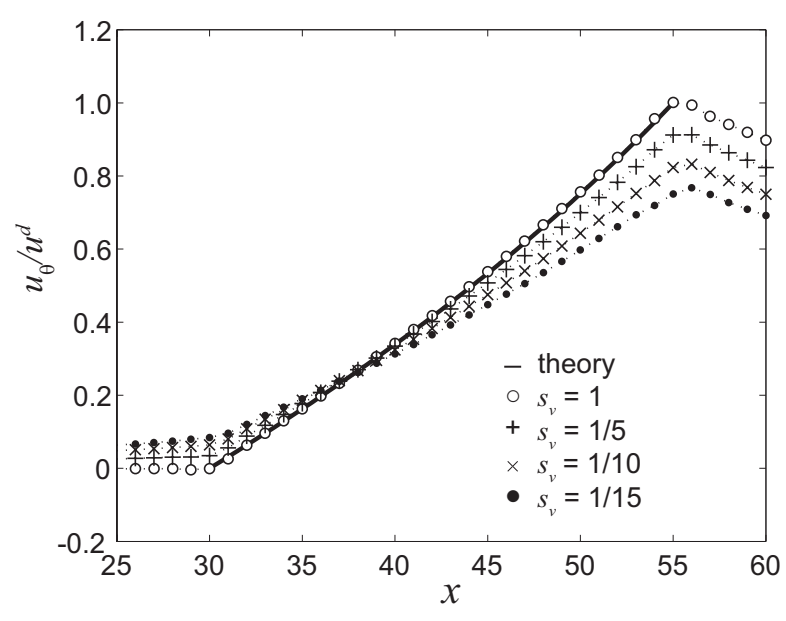

(a) MRT direct forcing method

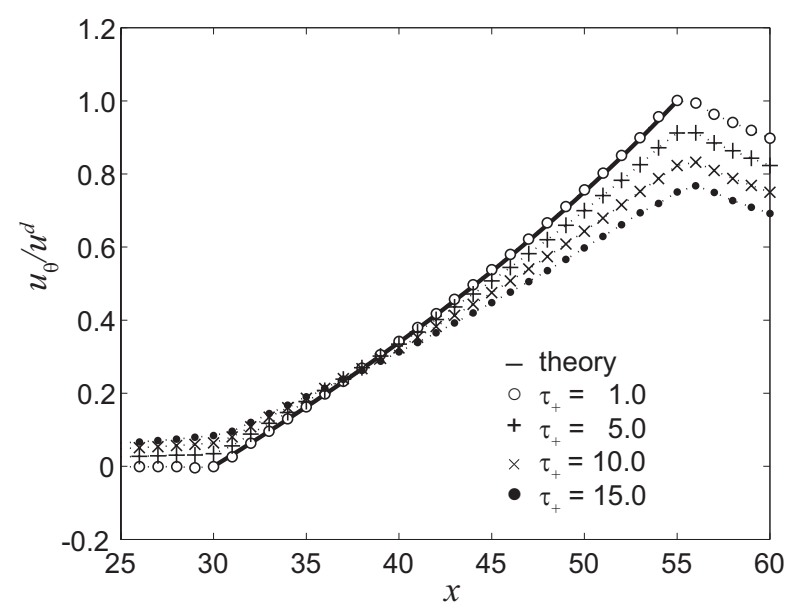

(c) TRT direct forcing method

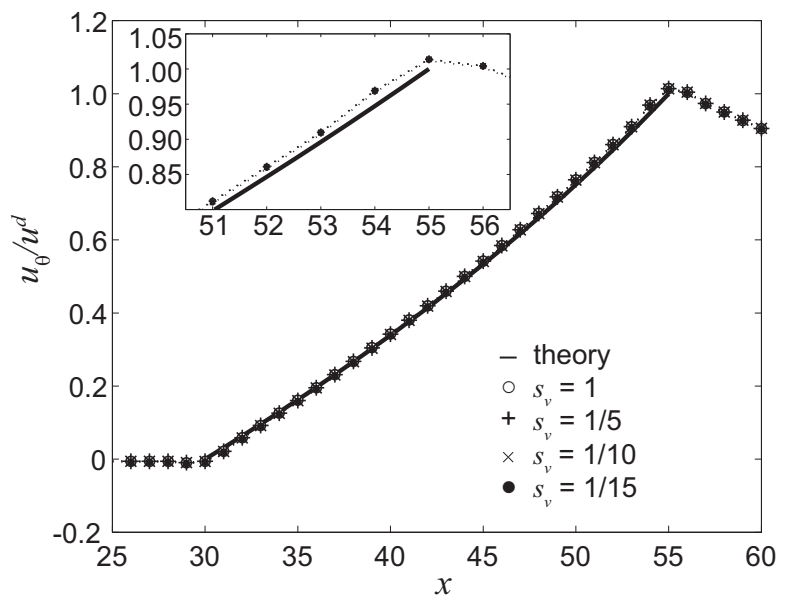

(b) MRT implicit correction method

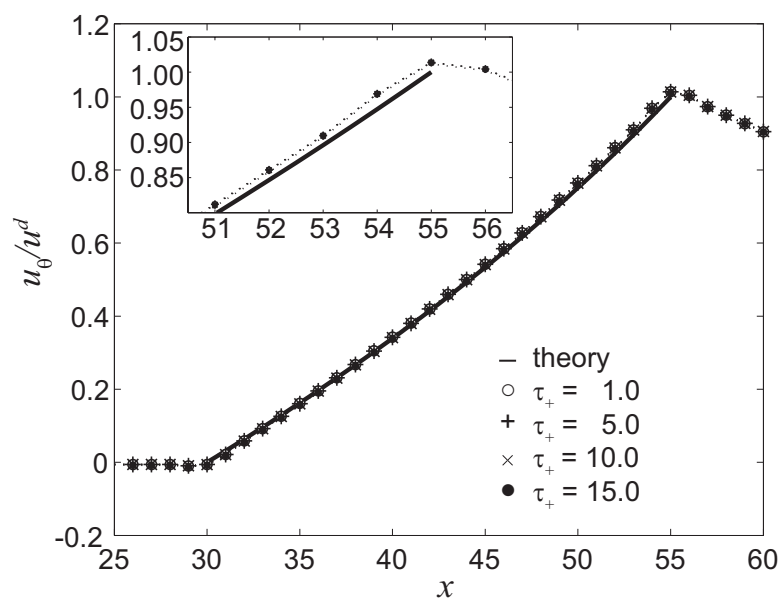

(d) TRT implicit correction method

FIG. 9. Velocity profiles calculated for the cylindrical Couette flows. The relaxation parameter $\tau_{+}$is varied as $1,5,10$, and 15 $\left(s_{v}=1 / \tau_{+}\right)$. The thick line indicates the exact velocity profile.

\section{Cylindrical Couette flow}

We examine the effectiveness of the TRT collision operator on the reduction of boundary slip in the simulation of the two-dimensional cylindrical Couette flow shown in Fig. 1(b). Figure 9 shows the profiles of the tangential velocity along $y=100 \delta_{x}$ at the horizontal centerline of the computational domain. The TRT model reduces the boundary slip and provides a smooth profile of the tangential velocity, as compared to the results shown in Figs. 2 and 3. In Figs. 9(a) and 9(c), the boundary error increases with the relaxation time for the direct forcing method. Although the numerical solutions exhibit a slight deviation from the exact solutions shown in Figs. 9(b) and 9(d), the application of the multiple relaxation collision operator to the implicit correction method successfully reduces the errors, as shown in Figs. 9(a) and $9(\mathrm{c})$.
Figure 10 shows the relative error defined by

$$
\text { Error }=\sqrt{\frac{\sum_{\mathbf{x} \in \text { fluid }}\left(u_{\theta}(\mathbf{x})-\hat{u}_{\theta}(\mathbf{x})\right)^{2}}{\sum_{\mathbf{x} \in \text { fluid }} \hat{u}_{\theta}(\mathbf{x})^{2}}},
$$

where fluid represents the region between the outer and inner rings shown in Fig. 1(b). The exact solution $\hat{u}_{\theta}$ is given by Eq. (44). Figure 10 shows the relative error versus the relaxation time. Although the multiplerelaxation-times scheme is applied to the direct forcing method, the relative error increases with the relaxation time in Fig. 10(a). Figure 10(b) shows that the accuracy of the implicit correction method with the TRT is the almost same as that of the MRT split forcing method. When the relaxation time is twenty, the error of the implicit correction method with multiple relaxation times becomes unacceptably large. As shown in Fig. 11(b), the numerical instability around the cylinders causes this degradation in accuracy of the IB-LBMs at $\tau_{+}=20$. In the calculation of the cylindrical Couette flows, the 


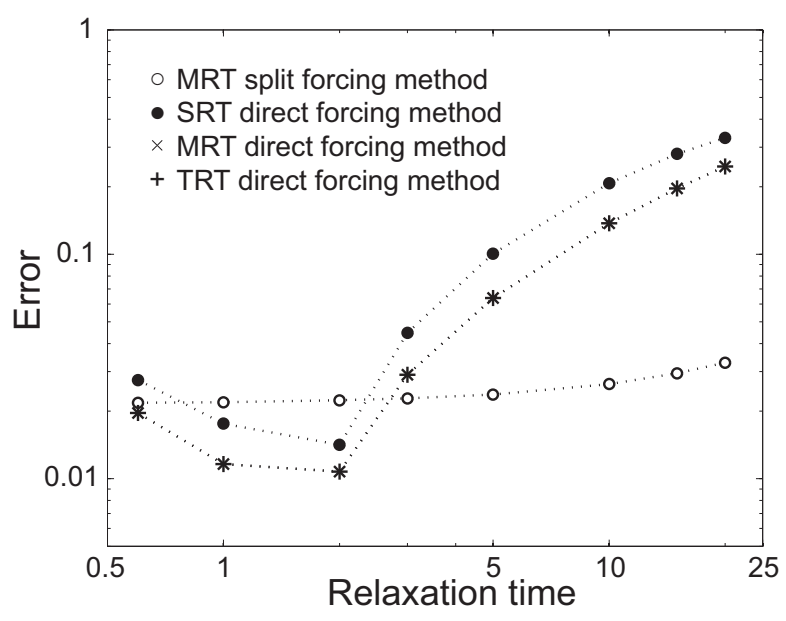

(a) Direct forcing method

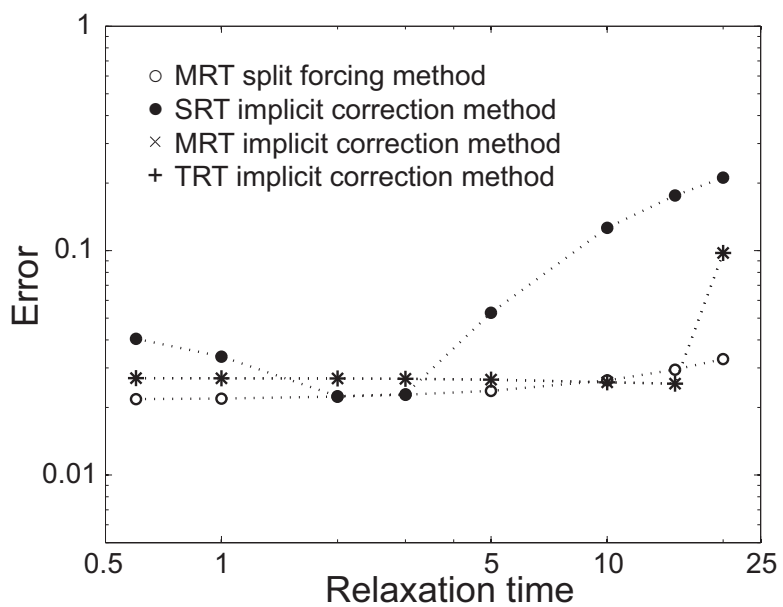

(b) Implicit correction method

FIG. 10. Relative error versus relaxation time in the calculation of the cylindrical Couette flows. The error calculated by the MRT split forcing method is added to all figures in order to compare the accuracy of the present results.

discrete area $\Delta s$ on the inner cylinder is approximately equal to that on the outer cylinder for all of the IB-LBMs. We set 282 Lagrangian points on the inner cylinder, and 439 points on the outer cylinder, which makes $\Delta s \approx \delta_{x}$.

In order to evaluate the numerical stability and accuracy of the TRT model, Ginzburg proposed the parameter $\Lambda=\left(2 \tau_{+}-1\right)\left(2 \tau_{-}-1\right) / 4$. For instance, $\Lambda=1 / 4$ gives the optimal stability for the TRT model, and $\Lambda=3 / 16$ gives the exact wall locations in the Poiseuille flow simulation using the bounce-back scheme [10, 12]. For the anisotropic advection-diffusion equation, $\Lambda=1 / 12$ and $\Lambda=1 / 6$ yield the best accuracy for the advection term and the diffusion term in the TRT model, respectively [19]. It is impossible to determine a unique parameter $\Lambda$ that yields the most accurate and most stable solution in the TRT collision model.

In the TRT implicit correction method, Eq. (42) yields

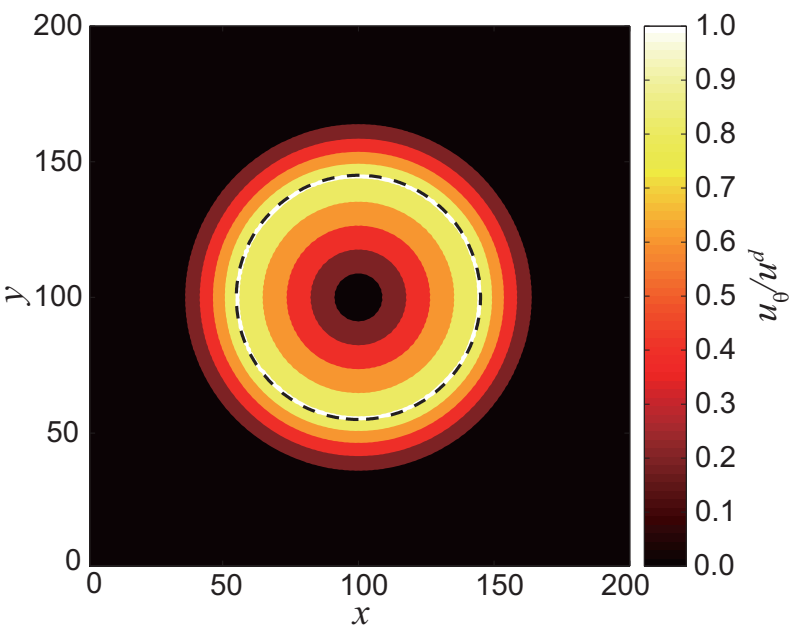

(a) $\tau_{+}=15$

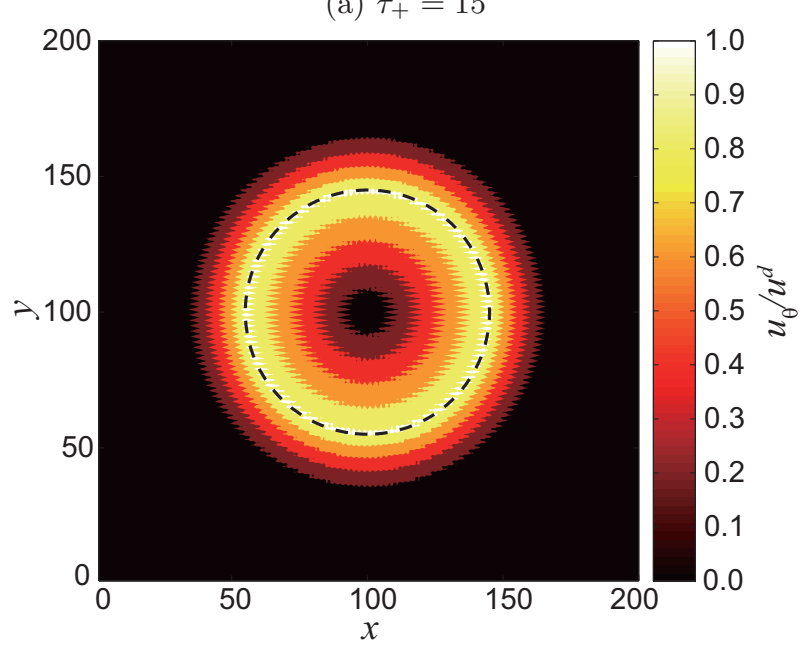

(b) $\tau_{+}=20$

FIG. 11. Tangential velocity profile in the cylindrical Couette flows as calculated by the TRT implicit correction method. The dashed circles indicates the position of the inner ring.

$\Lambda=9 / 8$ in order to reduce the boundary slip. In order to suppress the non-physical oscillations in the moving boundary problem, Yang et al. proposed the following smoothed three-point discrete delta function [20]:

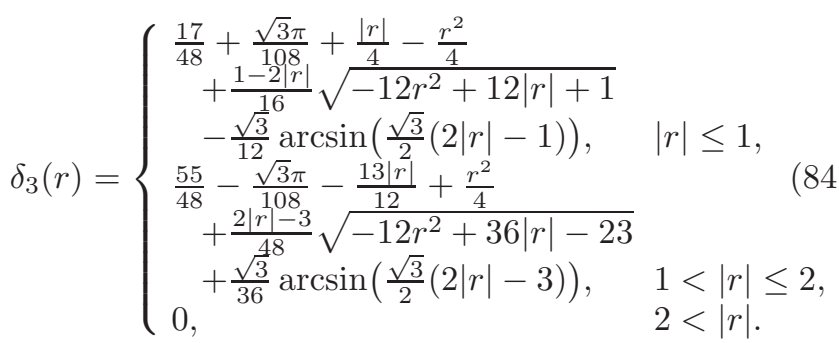

In order to reduce the boundary slip in the IB-LBM using Eq. (84), the following equation must be satisfied:

$$
\tau_{-}=\frac{4 \tau_{+}+4.7056}{8 \tau_{+}-4} .
$$

Equation (85) yields $\Lambda=6.7056 / 8$. Although the value 


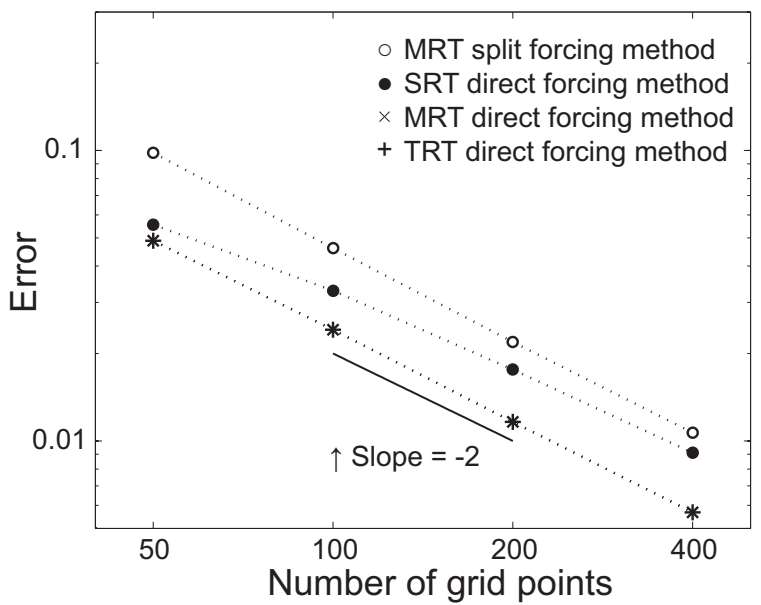

(a) Direct forcing method $\left(\tau_{+}=s_{v}=1\right.$.)

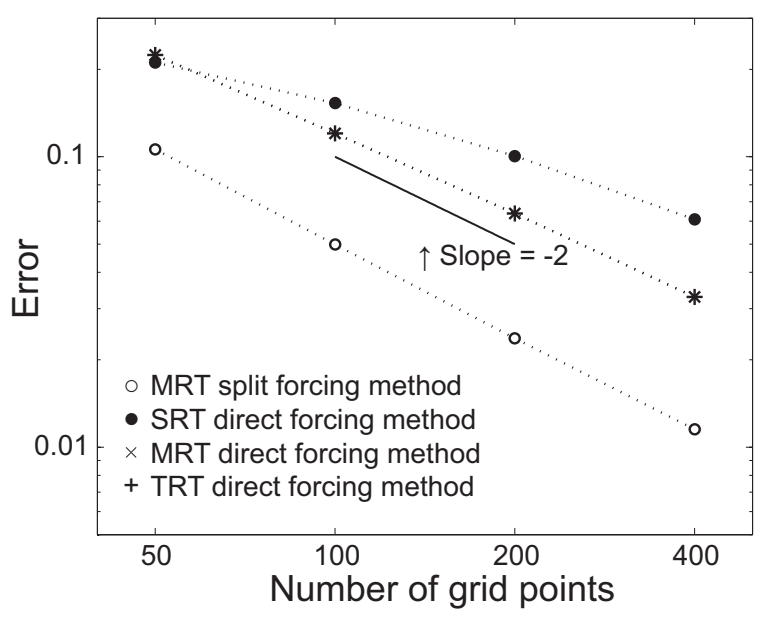

(c) Direct forcing method $\left(\tau_{+}=5, s_{v}=0.2\right.$.)

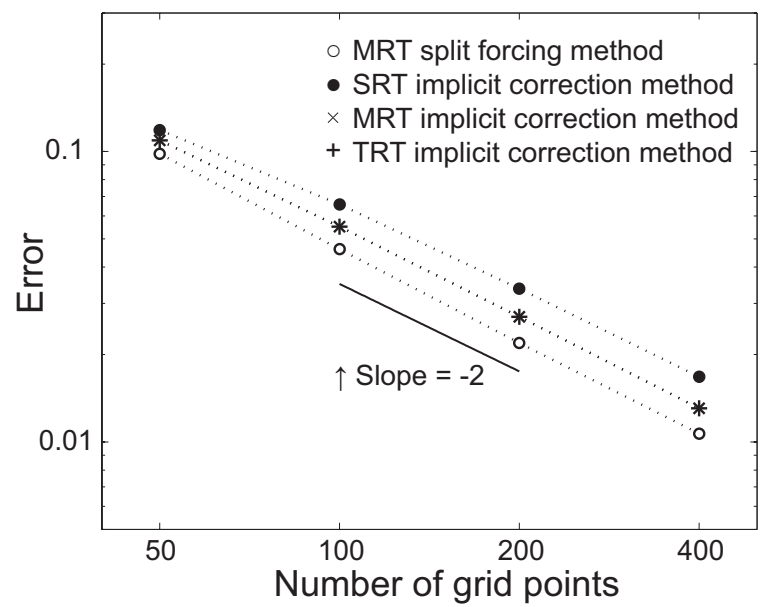

(b) Implicit correction method $\left(\tau_{+}=s_{v}=1\right.$.)

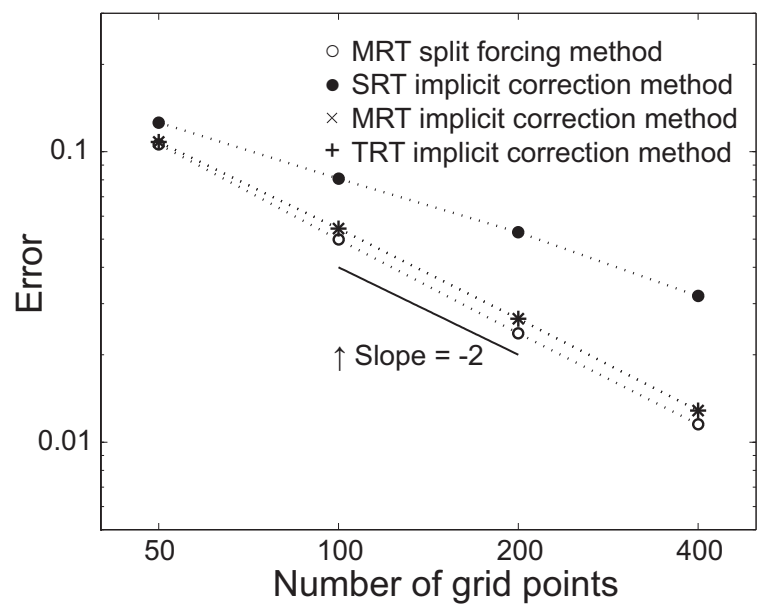

(d) Implicit correction method $\left(\tau_{+}=5, s_{v}=0.2\right.$.)

FIG. 12. Relative error versus the number of grid points. The relaxation times are set to be unity and five.

of $\Lambda$ affects the numerical stability, the value of $\Lambda$ depends on the discrete delta function for the boundary slip problem in the IB-LBMs. In order to prevent numerical instability, it will be necessary to investigate the adequate discrete area $\Delta s$, the parameter $\Lambda$, and the discrete delta function $\delta(r)$ for the TRT implicit correction method in the future.

Figure 12 shows the relation between the accuracy of the IB-LBMs and the number of grid points on a side of the square computational domain in the simulation of the cylindrical Couette flows. The IB-LBMs with the TRT exhibit second-order accuracy in space for both the direct forcing method and the implicit correction method. Figures $12(\mathrm{c})$ and $12(\mathrm{~d})$ show that the boundary slip caused by the use of the SRT model in the IB-LBM impairs the accuracy that the LBM intrinsically possesses. For all of the numerical results obtained in the two-dimensional stimulations, the IB-LBMs with TRT have exactly the same accuracy as the MRT collision models, while maintaining second-order accuracy in space.

\section{E. Boundary velocity error}

Although the TRT implicit correction method succeeds in reducing the boundary slip while maintaining secondorder accuracy in space, eliminating the difference between the boundary velocity $u_{j_{0}}$ on the Eulerian node and the desired velocity $u^{d}$ is difficult. We next investigate the boundary velocity error as computed by the TRT implicit correction method in the calculation of the symmetric shear flow. Figure 13 shows the boundary velocity $u_{b}$ on the Lagrangian node computed based on the results shown in Fig. 5. The solid line shows the analytical solution given by Eq. (58) for the direct forcing method. Although the boundary velocity $u_{b}$ and the desired velocity $u^{d}$ differ between the direct forcing method and the split forcing method [14], the implicit correction method makes $u_{b} / u^{d}=1$ for all relaxation times. For the direct forcing method, Eq. (8) leads to $u^{d}=u_{b}+G_{0} \delta_{t}$. The split forcing method obtains $u^{d}=u_{b}+\frac{1}{2} G_{0} \delta_{t}$ from 


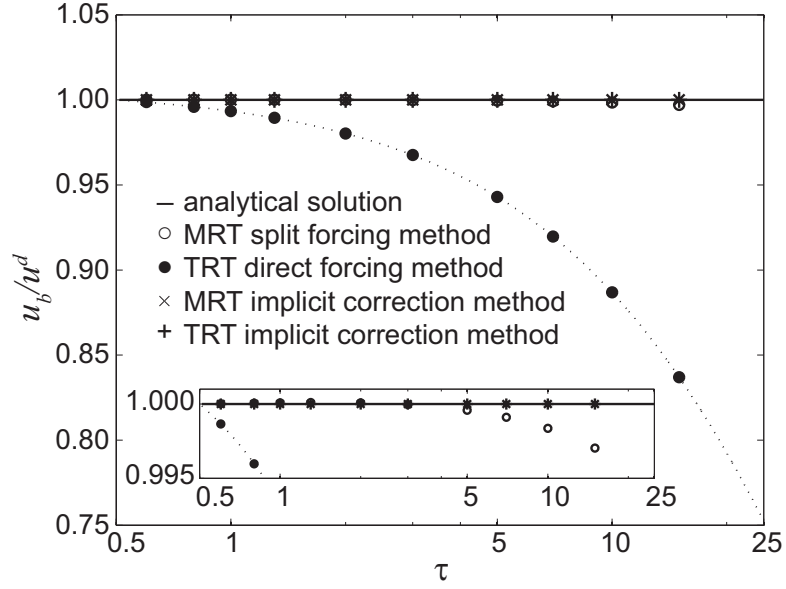

FIG. 13. Boundary velocity at the Lagrangian point in the calculation of the symmetric shear flows. These results were calculated based on the numerical velocity shown in Fig. 5. The horizontal axis indicates the relaxation time $\tau$, including $\tau_{+}$and $s_{v}$.

Eq. (32). Except in the case of $G_{0}=0, u^{d}=u_{b}+G_{0} \delta_{t}$ and $u^{d}=u_{b}+\frac{1}{2} G_{0} \delta_{t}$ do not give $u_{b}=u^{d}$. Since the implicit correction method solves the simultaneous equation given by Eq. (20) to make $u_{b}=u^{d}$, the result $u_{b} / u^{d}=1$ shown in Fig. 13 corresponds well with what is expected theoretically.

The velocity $u_{b}$ on the Lagrangian nodes is interpolated from the velocities on the Eulerian nodes by $u_{b}=\left(u_{j_{0}}+u_{j_{0}+1}\right) / 2$ in the symmetric shear flow. Since $u_{j_{0}}$ is larger than $u_{j_{0}+1}$, as shown in Fig. 5 , the velocity on the Eulerian node $u_{j_{0}}$ is larger than the desired velocity $u^{d}$ necessary to make $u_{b}$ equal to $u^{d}$ in the implicit correction method. Figure 14 shows the velocity profiles for the symmetric shear flow as calculated by the IBLBMs with the TRT using the following non-smoothed delta function:

$$
\delta(r)= \begin{cases}1 & |r|=0 \\ 0 & |r| \neq 0\end{cases}
$$

instead of Eq. (11). In Fig. 14(a), the TRT direct forcing method exhibits disagreement between the numerical and theoretical solutions at high relaxation time. The numerical error observed in the direct forcing method is due not only to the interpolation but also to the calculation of the body force shown by Eq. (8). Figure 14(b) shows that the TRT implicit correction method succeeds in making $u_{j 0}=u^{d}$ at any relaxation time without the boundary slip. The numerical results shown in Fig. 14 indicate that the smoothing technique of the delta function used in Eq. (9) causes the difference between $u_{j_{0}}$ and $u^{d}$ in the calculation of the IB-LBMs.

Equation (46) gives the analytical solution of the IBLBMs using Eq. (86) in the simulations of the symmetric

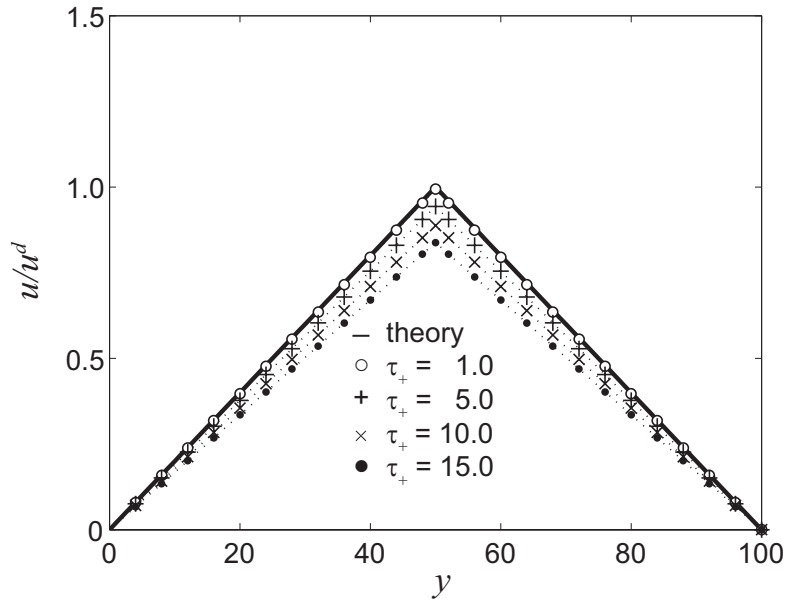

(a) TRT direct forcing method

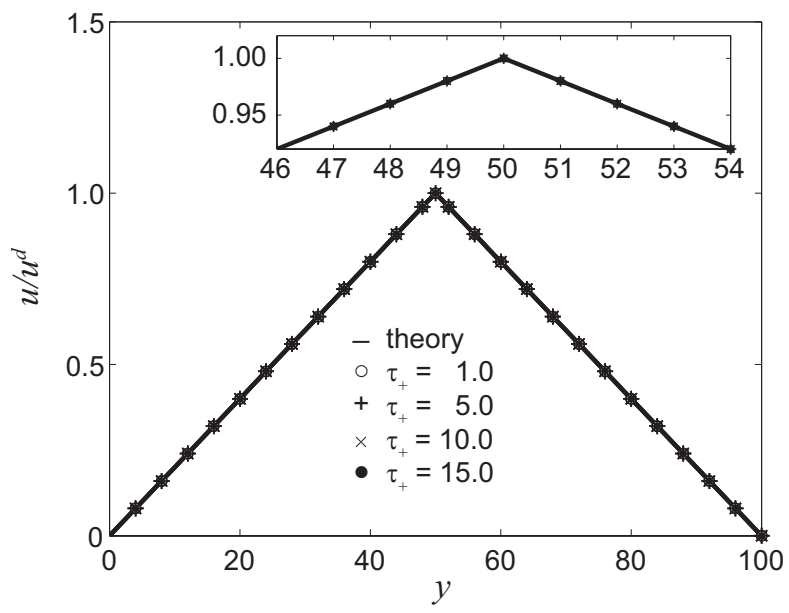

(b) TRT implicit correction method

FIG. 14. Velocity profiles of the symmetric shear flows, as calculated by the IB-LBMs with the TRT using the nonsmoothed delta function. The relaxation parameter $\tau_{+}$is varied as 1, 5, 10, and 15 . The thick line indicates the exact velocity profile.

shear flow:

$$
u_{j_{0}}=\frac{\delta_{x}^{2} G_{0}}{\nu}\left(\frac{h}{4}-\phi\right),
$$

and

$$
u^{s}=-\frac{\delta_{x}^{2} G_{0} \phi}{\nu} .
$$

When $\phi=0$, Eqs. (87) and (88) give $u_{j_{0}}=\bar{u}_{j_{0}}$ and $u^{s}=0$, respectively. The exact solution is given by $\bar{u}_{j_{0}}=$ $\left(h G_{0} \delta_{x}^{2}\right) /(4 \nu)$. Substituting $\phi=0$ into Eqs. (47) and (55), we obtain

$$
\tau_{-}=\frac{3 \tau_{+}}{4 \tau_{+}-2},
$$

for the direct forcing method, and

$$
\tau_{-}=\frac{4 \tau_{+}+1}{8 \tau_{+}-4},
$$




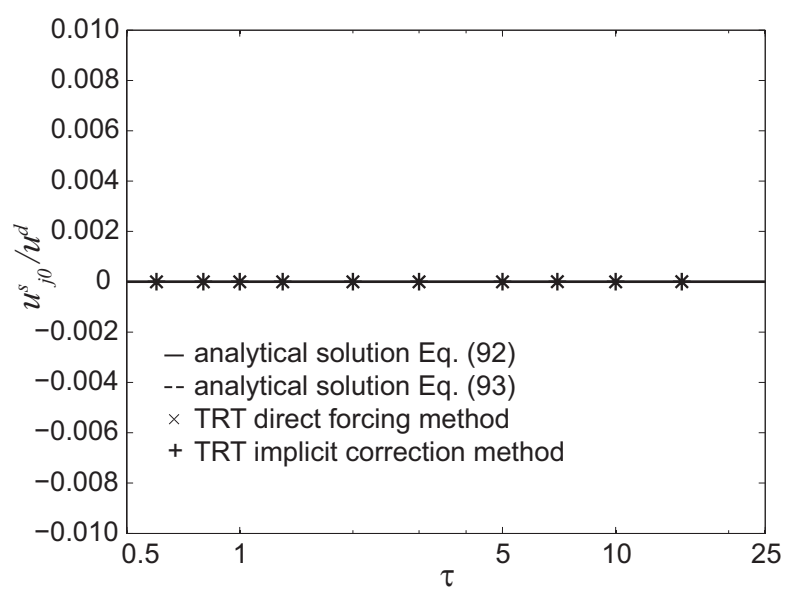

(a) Boundary slip velocity

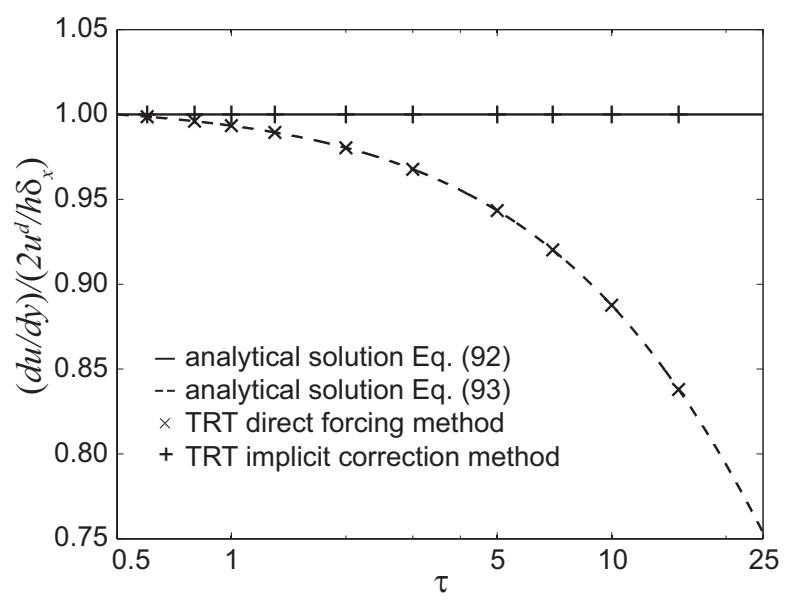

(b) Velocity gradient

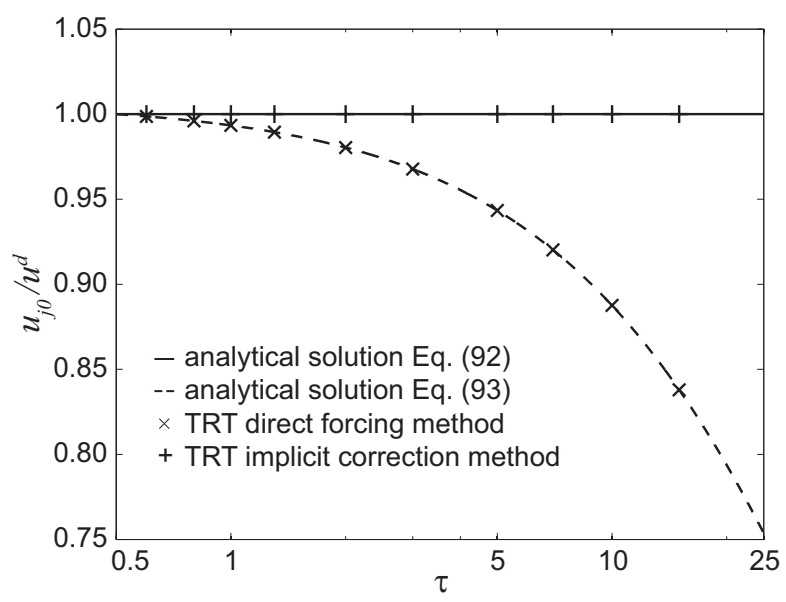

(c) Fluid velocity at the boundary

FIG. 15. Comparison of the analytical and the numerical solutions as calculated by the TRT direct forcing method and by the TRT implicit correction method using a non-smoothed delta function.

for the implicit correction method. When $\phi=0$, Eq. (46)
TABLE I. Comparison of drag coefficient, wake length, and separation angle as calculated by the TRT implicit correction method and in previous studies.

\begin{tabular}{clccc}
\hline \hline Re & References & $C_{d}$ & $2 L / D$ & $\theta_{s}$ \\
\hline 40 & Dennis and Chang [21] & 1.522 & 4.69 & 53.8 \\
\cline { 2 - 5 } & Nieuwstadt and Keller [22] & 1.550 & 4.357 & 53.34 \\
\cline { 2 - 5 } & He and Doolen [23] & 1.499 & 4.490 & 52.84 \\
\cline { 2 - 5 } & Present study & 1.568 & 4.640 & 50.38 \\
\hline 20 & Dennis and Chang [21] & 2.045 & 1.88 & 43.7 \\
\cline { 2 - 5 } & Nieuwstadt and Keller [22] & 2.053 & 1.786 & 43.37 \\
\cline { 2 - 5 } & He and Doolen [23] & 2.152 & 1.842 & 42.96 \\
\cline { 2 - 5 } & Present study & 2.104 & 1.900 & 40.78 \\
\hline \multirow{2}{*}{10} & Dennis and Chang [21] & 2.846 & 0.53 & 29.6 \\
\cline { 2 - 5 } & Nieuwstadt and Keller [22] & 2.828 & 0.434 & 27.96 \\
\cline { 2 - 5 } & He and Doolen [23] & 3.170 & 0.474 & 26.89 \\
\cline { 2 - 5 } & Present study & 2.920 & 0.520 & 26.36 \\
\hline \hline
\end{tabular}

yields

$$
0=\nu \frac{u_{j+1}-2 u_{j}+u_{j-1}}{\delta_{x}^{2}}+G_{j},
$$

which is the steady-state Navier-Stokes equation used in the calculation of the symmetric shear flows by the IBLBMs.

Finally, the analytical solutions are given by

$$
\frac{u_{j_{0}}^{s}}{u^{d}}=0, \quad \frac{\left(\frac{d u}{d y}\right)_{b u l k}}{\frac{2 u^{d}}{h \delta_{x}}}=\frac{u_{j_{0}}}{u^{d}}=1,
$$

for the TRT implicit correction method, and

$$
\frac{u_{j_{0}}^{s}}{u^{d}}=0, \quad \frac{\left(\frac{d u}{d y}\right)_{b u l k}}{\frac{2 u^{d}}{h \delta_{x}}}=\frac{u_{j_{0}}}{u^{d}}=\frac{\frac{h}{4}}{\frac{h}{4}+\frac{\tau_{+}}{3}-\frac{1}{6}},
$$

for the TRT direct forcing method.

Figure 15 compares the numerical and analytical solutions for the IB-LBMs obtained using the non-smoothed delta function shown by Eq. (86). As predicted by Eqs. (92) and (93), in contrast to the direct forcing method, the implicit correction method completely eliminates all numerical errors. The boundary velocity error $u_{j_{0}} / u^{d}$ is confirmed to be induced by the smoothed delta function used in the interpolation procedure. Since the positions of the Lagrangian grids are not equal to those of the Eulerian grids in the fluid flow simulation by the IB-LBMs, it is not possible to use the non-smoothed delta function. Although the boundary error due to the smooth approximation of the delta function is inevitable, the error decreases with the elongation of the characteristic length $h$ in the implicit correction method, as shown in Eqs. (71) and (73). 


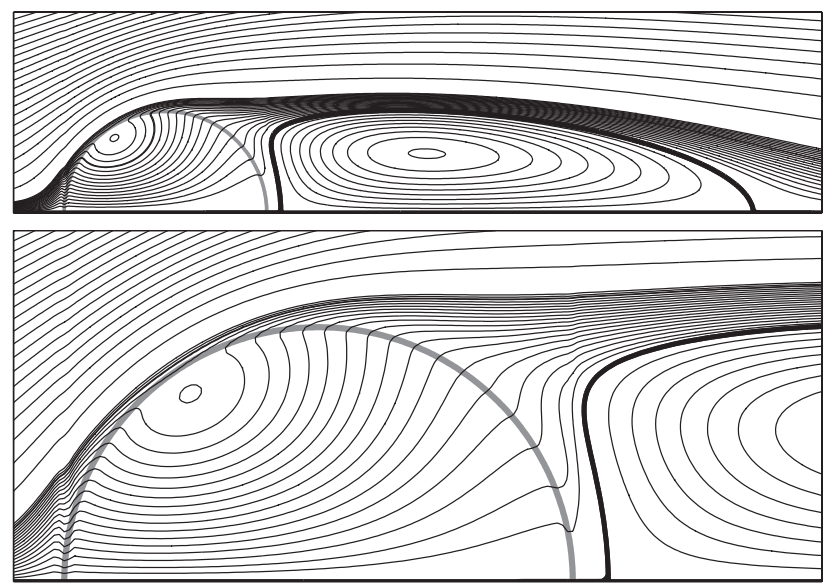

(a) SRT direct forcing method

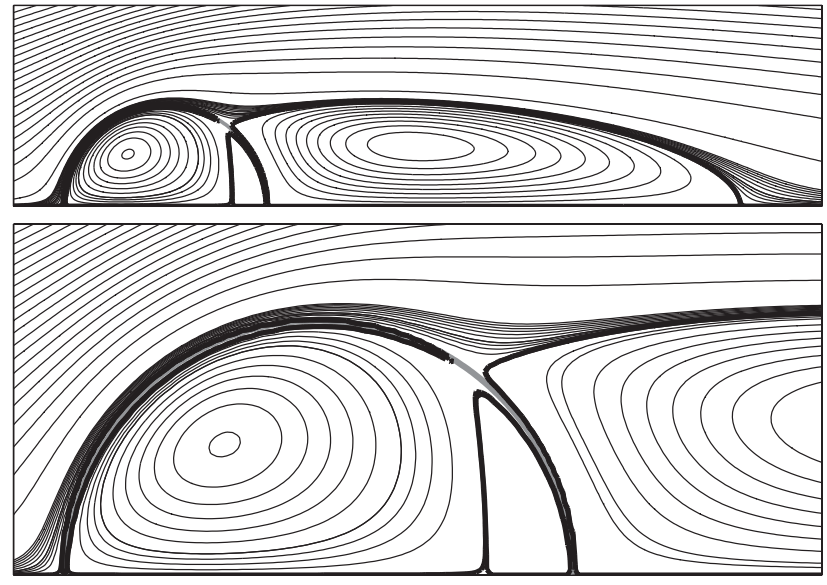

(c) SRT implicit correction method

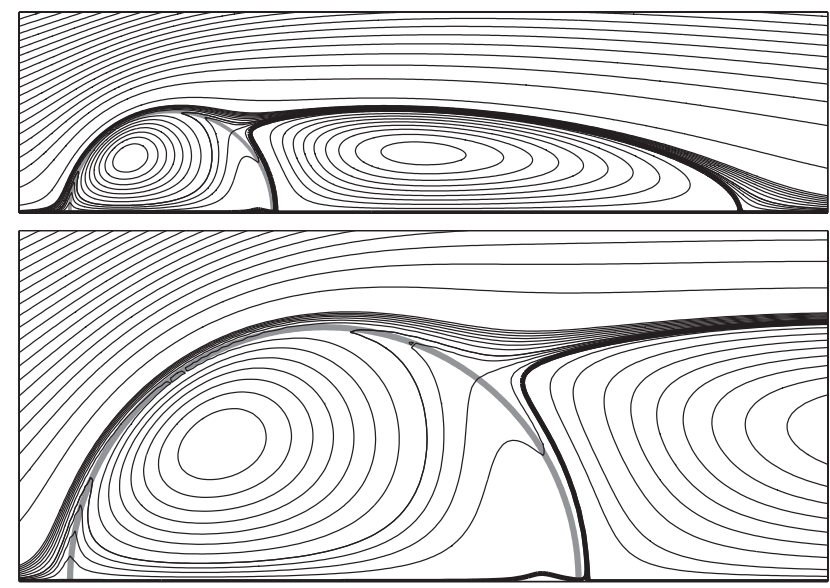

(b) MRT split forcing method

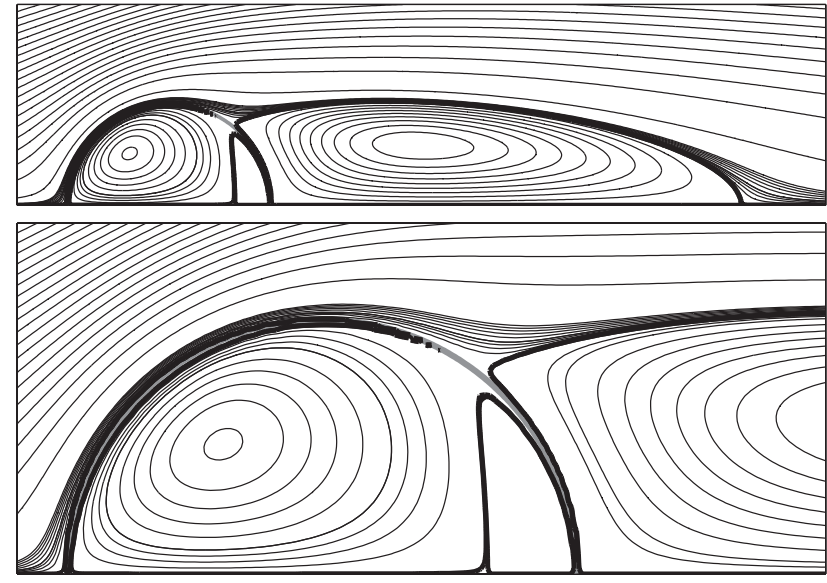

(d) TRT implicit correction method

FIG. 16. Streamlines for a flow past a circular cylinder at $R e=40$. The thick line indicates the zero streamline. The surface of the circular cylinder is indicated by the gray line. The relaxation time is equal to 1.25 .

\section{F. Flow past a circular cylinder}

The final test is the calculation of the fluid flow past a circular cylinder at $R e=u_{0} D / \nu=10,20$, and 40. Numerous theoretical, experimental, and numerical results are available for comparison to the present results. The diameter of the cylinder is given by $D=100 \delta_{x}$, and the characteristic free-stream velocity $u_{0}$ is equal to $0.1 c$. The cylinder is located at $(16 \mathrm{D}, 20 \mathrm{D})$ in the computational domain with $40 D \times 40 D$. The fluid density is taken as $\rho=1.0$. We use the smoothed delta function given by Eq. (11). In order to make $\Delta s \approx 0.125 \delta_{x}, 2,513$ Lagrangian points are set on the cylinder in the calculation by the SRT direct forcing method and by the MRT split forcing method. For $\Delta s \approx 2 \delta_{x}$, we use $\Delta s=100 \pi \delta_{x} / 157$ for the cylinder in the calculation by the implicit correction method. The relaxation times are equal to 1.25, 2.0, and 3.5 for $\operatorname{Re}=40,20$, and 10, respectively.

Figures 16, 17, and 18 show the streamlines in the half computational domain calculated by the IB-LBMs at $\operatorname{Re}=40,20$, and 10 , respectively. The thick solid line indicates the zero streamline. The gray semicircle indi- cates the surface of the circular cylinder. In Figs. 16(a), 17(a), and 18(a), the SRT direct forcing method shows the penetration of the streamline through the surface of the cylinder. The MRT split forcing method decreases the penetration of the streamline observed in the SRT direct forcing method. In Fig. 18(b), the recirculating eddy, however, does not sufficiently develop behind the cylinder due to the penetration of the streamline at $\mathrm{Re}=10$. As Wu et al. reported [6], the implicit correction method prevents the penetration of the streamlines into the solid body and produces recirculating eddies with adequate wake length and separation points. When the SRT is used in the implicit correction method, the streamline on the cylinder surface is not always zero in Fig. 18(c). As shown in Fig. 18(d), by reducing the velocity slip, the TRT implicit correction method removes the distortion of the streamline observed in Fig. 18(c).

The drag coefficient is defined as follows:

$$
C_{d}=\frac{F_{D}}{(1 / 2) \rho u_{0}^{2} D} .
$$




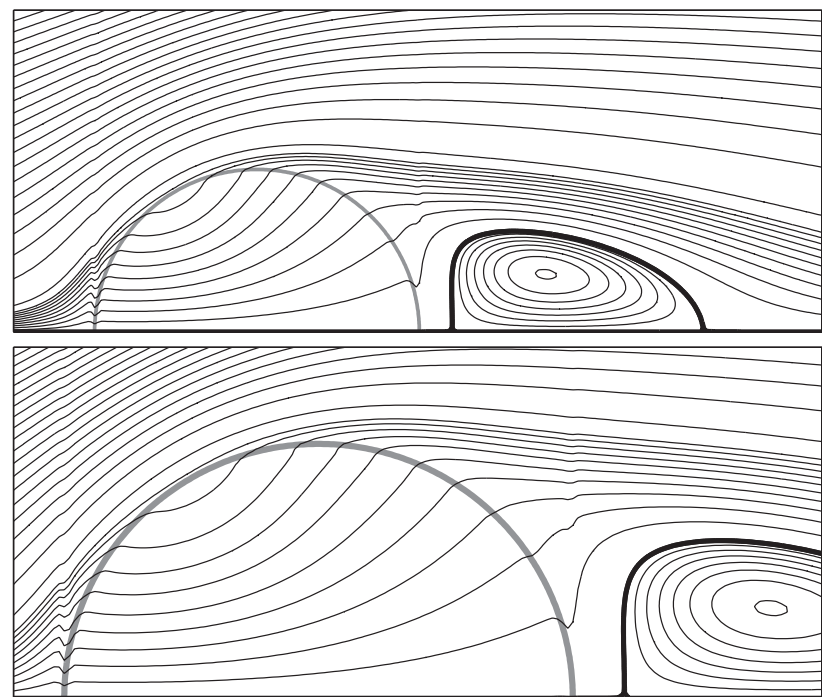

(a) SRT direct forcing method

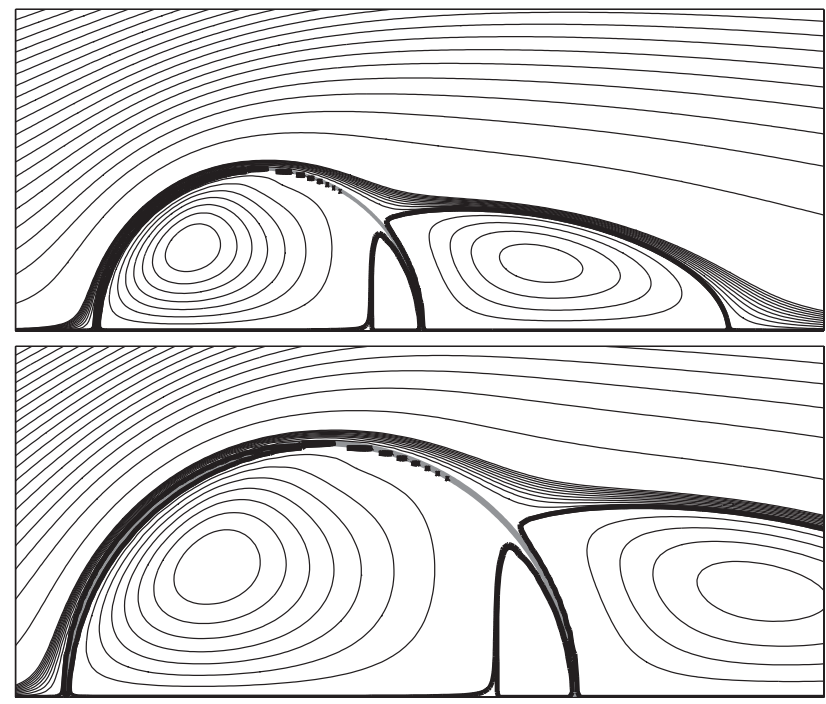

(c) SRT implicit correction method

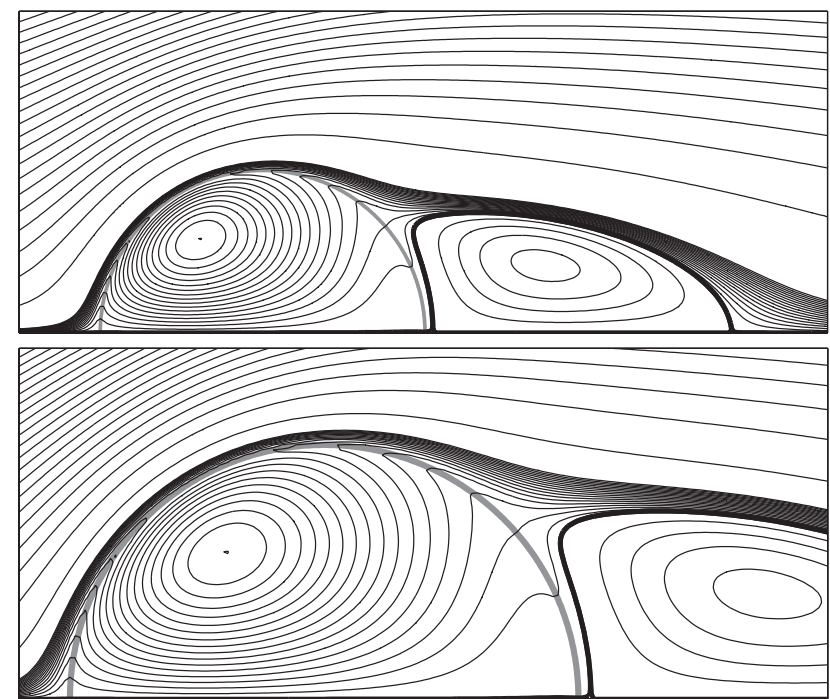

(b) MRT split forcing method

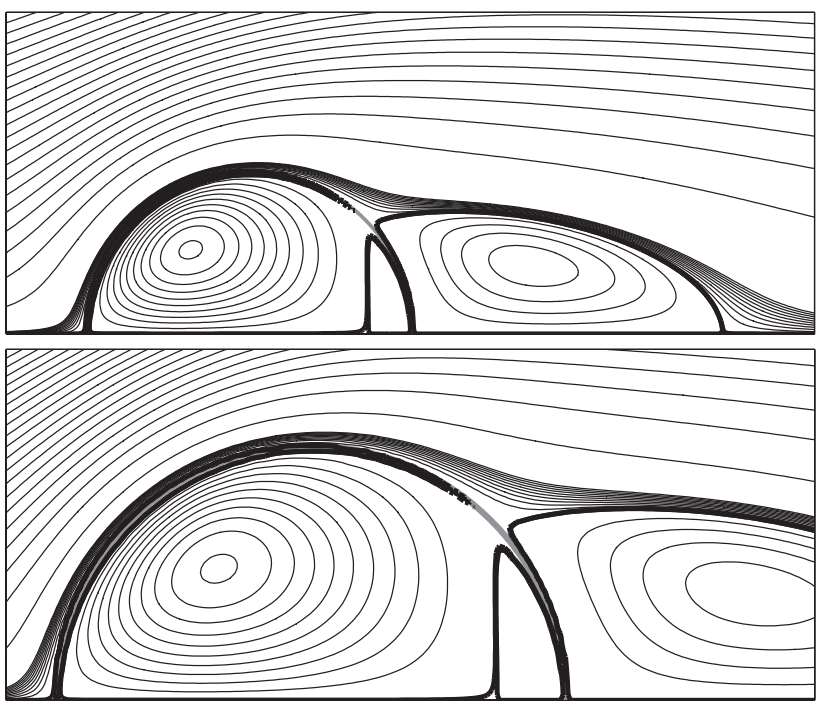

(d) TRT implicit correction method

FIG. 17. Streamlines for a flow past a circular cylinder at Re $=20$. The thick line indicates the zero streamline. The surface of the circular cylinder is indicated by the gray line. The relaxation time is equal to 2.0

Here, $F_{D}$ is the drag force calculated by

$$
F_{D}=-\int_{\Omega} G_{x} d x
$$

where $G_{x}$ is the $x$ component of the force density $\mathbf{G}$.

Table I shows the drag coefficient $C_{D}$, the wake length $L$ normalized with respect to the radius of the cylinder, and the separation angle $\theta_{s}$ in the calculation of the TRT implicit correction method. Dennis and Chang solved the governing equations using a finite difference method in the polar coordinate system [21]. Nieuwstadt and Keller numerically investigated the flow past a circular cylinder using the semi-analytical method with the stream function and the vorticity in a finite Fourier series [22]. He and Doolen used the lattice Boltzmann method extended to the general curvilinear coordinate system using the interpolated strategy [23]. Good agreement between the results of the proposed method and the results of previous methods is observed [21-23].

\section{CONCLUSION}

We analytically and numerically investigated the solutions calculated by the IB-LBM with the TRT for the symmetric shear flows and for the symmetric Poiseuille flow in order to derive the theoretical relation between two relaxation times so as to eliminate the boundary slip. The simple TRT collision operator reduced the boundary slip velocity occurring at the high relaxation time in the IB-LBM calculation as effectively as the MRT collision operator. The IB-LBM with the multiple relaxation 


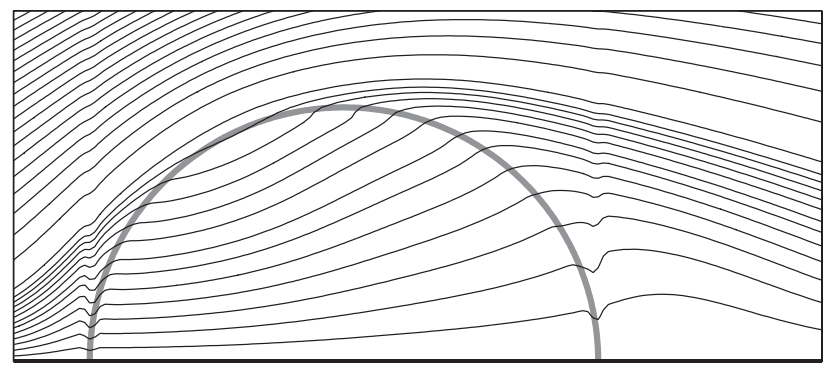

(a) SRT direct forcing method

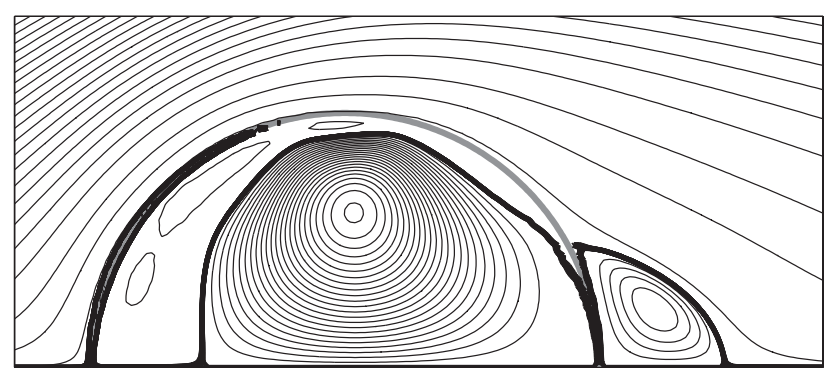

(c) SRT implicit correction method

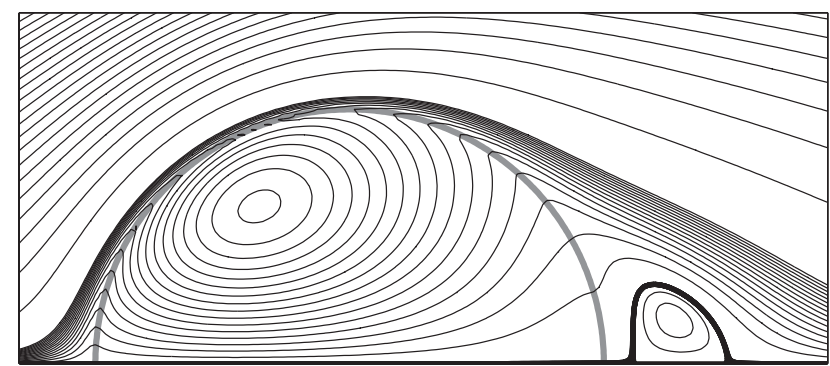

(b) MRT split forcing method

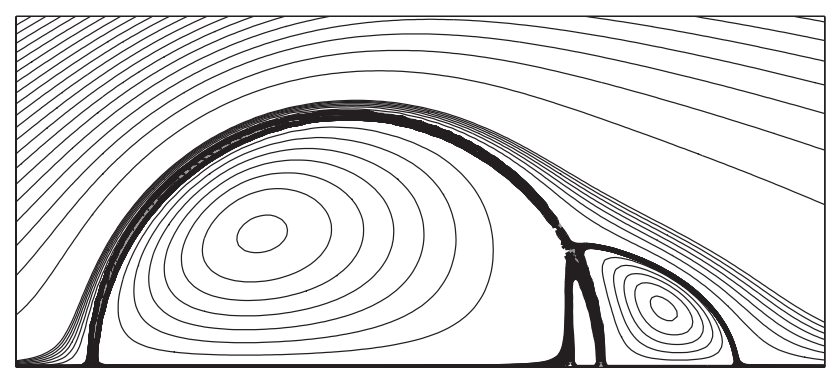

(d) TRT implicit correction method

FIG. 18. Streamlines for a flow past a circular cylinder at $R e=10$. The thick line indicates the zero streamline. The surface of the circular cylinder is indicated by the gray line. The relaxation time is equal to 3.5

times collision operator exhibited second-order accuracy in space for the convergence rate in the calculation of the cylindrical Couette flows. Lu et al. reported one problem, which is the disagreement between the boundary velocity on the Eulerian grid and the desired velocity [14]. We demonstrated that this problem was induced by the smoothing technique in the delta function used in the interpolation procedure. The error in the calculation of the IB-LBMs using the smoothed delta function is inevitable. In the calculation of the flow past a circular cylinder, the TRT implicit correction method prevented the streamline from penetrating through the solid surface as well as the velocity distortion due to the boundary slip, which allowed the recirculating wakes behind the cylinder to be reasonably computed.

To yield the transfer matrix $M$ satisfying $|R\rangle=M|f\rangle$, the multi-relaxation time D3Q15 model requires the 15 components in momentum vector $|R\rangle$ corresponding to the fifteen distribution functions $|f\rangle$ [24]. For the D3Q15 and D3Q19 models, the TRT collision model can be easily formulated using only the distribution function $f_{k}$ and the counterpart $f_{\bar{k}}$ with the opposite direction to $f_{k}$ regardless of the number of the distribution functions. The TRT collision operator is better suited than the MRT collision operator to the three-dimensional problem for reducing the boundary slip in the IB-LBM.

For the application of the multi-block approach in the IB-LBM, the relaxation times $\tau_{f}$ on fine gird and $\tau_{c}$ on the coarse grid obey the relation, $\tau_{f}=n\left(\tau_{c}-0.5\right)+$ 0.5 , in order to maintain a consistent viscosity for the any grid size [25]. Here, $n=\delta_{x_{c}} / \delta_{x_{f}}$ is the ratio of the lattice space between the coarse and fine grids. Since the numerical solution is not influenced by the relaxation time, the TRT implicit correction method can be used to extend the IB-LBMs to the multi-block framework.

\section{ACKNOWLEDGMENTS}

The present study was supported by a Grant-in-Aid for Scientific Research (No. 23560190) from the Ministry of Education, Science, and Culture of Japan. The authors would like to thank Prof. R. Takahashi, Prof. E. Takegosi, Prof. K. Okui, and Prof. A. Abe for their helpful suggestions.

\section{Appendix A: ANALYTICAL SOLUTIONS FOR SYMMETRIC SHEAR FLOWS}

Since $f_{k}\left(\mathbf{x}+\mathbf{e}_{k} \delta_{t}, t+\delta_{t}\right)=f_{k}(\mathbf{x}, t)$ for $k=1$ and 3 in a steady state, Eqs. (41) and (45) yields

$$
\begin{aligned}
0=- & \frac{1}{2}\left(\frac{1}{\tau_{+}}+\frac{1}{\tau_{-}}\right) f_{1}^{j}-\frac{1}{2}\left(\frac{1}{\tau_{+}}-\frac{1}{\tau_{-}}\right) f_{3}^{j} \\
+\frac{\rho}{9 \tau_{+}} & \left(1+\frac{3 u_{j}^{2}}{c^{2}}\right)+\delta_{t}\left(1-\frac{1}{2 \tau_{+}}\right) \frac{2 \rho u G_{j}}{3 c^{2}} \\
+ & \frac{\rho u_{j}}{3 c \tau_{-}}+\delta_{t}\left(1-\frac{1}{2 \tau_{-}}\right) \frac{\rho G_{j}}{3 c}
\end{aligned}
$$




$$
\begin{gathered}
0=-\frac{1}{2}\left(\frac{1}{\tau_{+}}+\frac{1}{\tau_{-}}\right) f_{3}^{j}-\frac{1}{2}\left(\frac{1}{\tau_{+}}-\frac{1}{\tau_{-}}\right) f_{1}^{j} \\
+\frac{\rho}{9 \tau_{+}}\left(1+\frac{3 u_{j}^{2}}{c^{2}}\right)+\delta_{t}\left(1-\frac{1}{2 \tau_{+}}\right) \frac{2 \rho u G_{j}}{3 c^{2}} \\
-\frac{\rho u_{j}}{3 c \tau_{-}}-\delta_{t}\left(1-\frac{1}{2 \tau_{-}}\right) \frac{\rho G_{j}}{3 c}
\end{gathered}
$$

for the TRT implicit correction method. In order to indicate the position along the $y$-axis, we use the subscript $j$ in for $f_{k}, u$, and $G$. Subtraction of Eq. (A1) from Eq. (A2) gives

$$
f_{1}^{j}-f_{3}^{j}=\frac{2 \rho}{3 c}\left\{u_{j}+\delta_{t}\left(\tau_{-}-\frac{1}{2}\right) G_{j}\right\} .
$$

According to Eqs. (4), (29), (34), (40), and (41) in the steady state, we have

$$
\begin{aligned}
& f_{5}^{j}=\left\{1-\frac{1}{2}\left(\frac{1}{\tau_{+}}+\frac{1}{\tau_{-}}\right)\right\} f_{5}^{j-1}-\frac{1}{2}\left(\frac{1}{\tau_{+}}-\frac{1}{\tau_{-}}\right) f_{7}^{j-1} \\
& +\frac{\rho}{36 \tau_{+}}\left(1+\frac{3 u_{j-1}^{2}}{c^{2}}\right)+\delta_{t}\left(1-\frac{1}{2 \tau_{+}}\right) \frac{\rho G_{j-1} u_{j-1}}{6 c^{2}} \\
& +\frac{\rho u_{j-1}}{12 c \tau_{-}}+\delta_{t}\left(1-\frac{1}{2 \tau_{-}}\right) \frac{\rho G_{j-1}}{12 c}, \\
& f_{6}^{j}=\left\{1-\frac{1}{2}\left(\frac{1}{\tau_{+}}+\frac{1}{\tau_{-}}\right)\right\} f_{6}^{j-1}-\frac{1}{2}\left(\frac{1}{\tau_{+}}-\frac{1}{\tau_{-}}\right) f_{8}^{j-1} \\
& +\frac{\rho}{36 \tau_{+}}\left(1+\frac{3 u_{j-1}^{2}}{c^{2}}\right)+\delta_{t}\left(1-\frac{1}{2 \tau_{+}}\right) \frac{\rho G_{j-1} u_{j-1}}{6 c^{2}} \\
& -\frac{\rho u_{j-1}}{12 c \tau_{-}}-\delta_{t}\left(1-\frac{1}{2 \tau_{-}}\right) \frac{\rho G_{j-1}}{12 c}, \\
& f_{7}^{j}=\left\{1-\frac{1}{2}\left(\frac{1}{\tau_{+}}+\frac{1}{\tau_{-}}\right)\right\} f_{7}^{j+1}-\frac{1}{2}\left(\frac{1}{\tau_{+}}-\frac{1}{\tau_{-}}\right) f_{5}^{j+1} \\
& +\frac{\rho}{36 \tau_{+}}\left(1+\frac{3 u_{j+1}^{2}}{c^{2}}\right)+\delta_{t}\left(1-\frac{1}{2 \tau_{+}}\right) \frac{\rho G_{j+1} u_{j+1}}{6 c^{2}} \\
& -\frac{\rho u_{j+1}}{12 c \tau_{-}}-\delta_{t}\left(1-\frac{1}{2 \tau_{-}}\right) \frac{\rho G_{j+1}}{12 c}, \\
& f_{8}^{j}=\left\{1-\frac{1}{2}\left(\frac{1}{\tau_{+}}+\frac{1}{\tau_{-}}\right)\right\} f_{8}^{j+1}-\frac{1}{2}\left(\frac{1}{\tau_{+}}-\frac{1}{\tau_{-}}\right) f_{6}^{j+1} \\
& +\frac{\rho}{36 \tau_{+}}\left(1+\frac{3 u_{j+1}^{2}}{c^{2}}\right)+\delta_{t}\left(1-\frac{1}{2 \tau_{+}}\right) \frac{\rho G_{j+1} u_{j+1}}{6 c^{2}} \\
& +\frac{\rho u_{j+1}}{12 c \tau_{-}}+\delta_{t}\left(1-\frac{1}{2 \tau_{-}}\right) \frac{\rho G_{j+1}}{12 c} .
\end{aligned}
$$

Using the momentum density in the $x$ direction given by Eq. (14), we obtain:

$$
\rho u=c\left(f_{1}-f_{3}+f_{5}-f_{6}-f_{7}+f_{8}\right)+\frac{\delta_{t} \rho G}{2} .
$$

Substituting Eqs. (A3) through (A7) into Eq. (A8) at the two positions $j \pm 1$ yields

$$
\begin{array}{r}
\left(2 \tau_{+} \tau_{-}-\tau_{+}-\tau_{-}+1\right)\left(f_{5}^{j}-f_{6}^{j}-f_{7}^{j}+f_{8}^{j}\right)= \\
\frac{\rho}{c}\left\{-\frac{\tau_{+}-1}{3} u_{j}+\frac{\tau_{-}\left(2 \tau_{+}-1\right)}{6}\left(u_{j+1}+u_{j-1}\right)\right. \\
+\frac{\tau_{-}\left(1+4 \tau_{+}+4 \tau_{-}-8 \tau_{+} \tau_{-}\right)}{12} \delta_{t}\left(G_{j+1}+G_{j-1}\right) \\
\left.-\frac{\left(2 \tau_{-}-1\right)\left(\tau_{+}-1\right)}{6} \delta_{t} G_{j}\right\} .
\end{array}
$$

Substituting Eqs. (A3) and (A9) into Eq. (A8) at position $j$, and considering Eq. (36), we obtain Eqs. (46) and (47).

When the total boundary force is given by $\mathbf{G}\left(\mathbf{x}_{\mathbf{b}}\right)=$ $\left(G_{0}, 0\right)$, Eqs. (10) and (12) yield

$$
G_{j_{0}}=\frac{G_{0}}{2}, \quad G_{j_{0} \pm 1}=\frac{G_{0}}{4}, \quad G_{\left|j-j_{0}\right| \geq 2}=0
$$

where $j_{0}$ denotes the position of the moving surface. Due to the symmetry of the flow, we obtain

$$
u_{j_{0}+1}=u_{j_{0}-1} \text {. }
$$

Substitution of Eqs. (A10) and (A11) into Eq. (46) gives the velocity difference in the immersed boundary layer (IBL):

$$
\begin{gathered}
u_{j_{0}}-u_{j_{0}+1}=\frac{G_{0} \delta_{x}^{2}}{\nu}\left(\frac{1}{4}-\frac{\phi}{4}\right), \\
u_{j_{0}+1}-u_{j_{0}+2}=\frac{G_{0} \delta_{x}^{2}}{\nu}\left(\frac{1}{2}-\frac{\phi}{4}\right) .
\end{gathered}
$$

Since the body force does not spread beyond the IBL, the velocity gradient in the bulk fluid is constant.

$$
u_{j-1}-u_{j}=\frac{G_{0} \delta_{x}^{2}}{2 \nu}, \quad \text { for } \quad j>j_{0}+2 .
$$

Equation (A14) indicates that the force applied at the boundary induces the bulk velocity gradient given by Eq. (66).

Since the velocity is zero on the center plane between the two plates, Eq. (66) gives the exact solution for the velocity at $j_{0}$ :

$$
\bar{u}_{j_{0}}=\frac{G_{0} \delta_{x}^{2}}{2 \nu} \frac{h}{2} .
$$

Equation (A14) gives the velocity at $j_{0}+2$ beyond the IBL as follows:

$$
u_{j_{0}+2}=\frac{G_{0} \delta_{x}^{2}}{2 \nu}\left(\frac{h}{2}-2\right) .
$$

Combining Eqs. (A12), (A13), and (A16), we have

$$
u_{j_{0}}=\frac{G_{0} \delta_{x}^{2}}{\nu}\left(\frac{h}{4}-\frac{1}{4}-\frac{\phi}{2}\right)
$$

and

$$
u_{j_{0} \pm 1}=\frac{G_{0} \delta_{x}^{2}}{\nu}\left(\frac{h}{4}-\frac{1}{2}-\frac{\phi}{4}\right) .
$$


Subtracting Eq. (A15) from Eq. (A17), we obtain the artificial slip velocity as

$$
u_{j_{0}}^{s}=u_{j_{0}}-\bar{u}_{j_{0}}=\frac{G_{0} \delta_{x}^{2}}{\nu}\left(-\frac{1}{4}-\frac{\phi}{2}\right) .
$$

Substituting Eq. (47) into Eq. (A19) yields

$$
u_{j_{0}}^{s}=\frac{G_{0} \delta_{x}^{2}}{\nu} \cdot \frac{8 \tau_{+} \tau_{-}-4 \tau_{+}-4 \tau_{-}-7}{24},
$$

from which we can deduce the following relation between $\tau_{-}$and $\tau_{+}$:

$$
\tau_{-}=\frac{4 \tau_{+}+7}{8 \tau_{+}-4}
$$

so that $u_{j_{0}}^{s}=0$. The boundary velocity $u_{b}=u\left(\mathbf{x}_{b}\right)$ is given by Eq. (9) as follows:

$$
u_{b}=\frac{u_{j_{0}}}{2}+\frac{u_{j_{0}+1}}{4}+\frac{u_{j_{0}-1}}{4} .
$$

Substituting Eqs. (A17) and (A18) into Eq. (A22), we obtain

$$
u_{b}=\frac{G_{0} \delta_{x}^{2}}{\nu}\left(\frac{h}{4}-\frac{3}{8}-\frac{3 \phi}{8}\right) .
$$

The desired velocity $u^{d}$ is equal to $u_{b}$ in the implicit correction method. From Eqs. (A17), (A19), and (A23), we obtain the analytical solutions given by Eqs. (48), (49), and (50).

For the TRT direct forcing method, substitution of Eq. (A23) into Eq. (8) yields

$$
u^{d}=u_{b}+\delta_{t} G_{0}=\frac{G_{0} \delta_{x}^{2}}{\nu}\left(\frac{h}{4}-\frac{3}{8}-\frac{3 \phi}{8}+\frac{\delta_{t} \nu}{\delta_{x}^{2}}\right) .
$$

Equations (36), (55), (A17), (A19), and (A24) give the analytical solutions given by Eqs. (56), (57), and (58) for the TRT direct forcing method.

For the MRT direct forcing method, Eq. (38) yields

$$
\begin{gathered}
f_{1}^{j}-f_{3}^{j}=\frac{\left(f_{5}^{j}-f_{6}^{j}-f_{7}^{j}+f_{8}^{j}\right)}{2}+\frac{\rho u_{j}}{2 c}+\frac{\delta_{t} \rho G_{j}}{c s_{q}}, \\
f_{5}^{j}-f_{6}^{j}=\left(1-\frac{s_{v}}{2}\right)\left(f_{5}^{j-1}-f_{6}^{j-1}\right) \\
-\frac{s_{v}}{2}\left(f_{7}^{j-1}-f_{8}^{j-1}\right)+\frac{\delta_{t} \rho G_{j-1}}{2 c}, \\
f_{8}^{j}-f_{7}^{j}=\left(1-\frac{s_{v}}{2}\right)\left(f_{8}^{j+1}-f_{7}^{j+1}\right) \\
+\frac{s_{v}}{2}\left(f_{5}^{j+1}-f_{6}^{j+1}\right)+\frac{\delta_{t} \rho G_{j+1}}{2 c} .
\end{gathered}
$$

for the symmetric shear flows. Equations (A25) through (A27) and the definition of momentum,

$$
\rho u=c\left(f_{1}-f_{3}+f_{5}-f_{6}-f_{7}+f_{8}\right),
$$

at the two positions $j \pm 1$ give

$$
\begin{array}{r}
f_{5}^{j}-f_{6}^{j}-f_{7}^{j}+f_{8}^{j}=\frac{\rho}{c}\left\{\frac{1}{6}\left(u_{j+1}+u_{j-1}\right)\right. \\
\left.-\frac{1-s_{v}}{2-s_{v}} \delta_{t} G_{j}+\frac{2 s_{v}+3 s_{q}-4}{6 s_{q}\left(2-s_{v}\right)} \delta_{t}\left(G_{j+1}+G_{j-1}\right)\right\} .
\end{array}
$$

Substituting Eqs. (A25) and (A29) into Eq. (A28) at the position $j$ and considering Eq. (30), we obtain Eq. (59) for the MRT direct forcing method.

\section{Appendix B: ANALYTICAL SOLUTIONS FOR SYMMETRIC POISEUILLE FLOWS}

In the Poiseuille flow, the constant body force $F$ is added to $G_{j}$ in Eq. (A10):

$$
G_{j}=\left\{\begin{array}{ll}
G_{0} / 2+F, & j=j_{0} \\
G_{0} / 4+F, & j=j_{0} \pm 1 \\
F, & \left|j-j_{0}\right|>1
\end{array},\right.
$$

where $F$ is the body force along the $x$-axis in Fig. 1(a). Substitution of Eqs. (A11) and (B1) into Eq. (46) gives the velocity differences between two adjacent Eulerian nodes:

$$
\begin{gathered}
u_{j_{0}}-u_{j_{0}+1}=\frac{\delta_{x}^{2}}{\nu}\left(\frac{G_{0}(1-\phi)}{4}+\frac{F}{2}\right), \\
u_{j_{0}+1}-u_{j_{0}+2}=\frac{\delta_{x}^{2}}{\nu}\left(\frac{G_{0}(2-\phi)}{4}+\frac{3 F}{2}\right) .
\end{gathered}
$$

As shown in Eq. (B1), the boundary force $G_{0}$ is zero beyond the IBL, the velocity difference is given by

$$
\begin{array}{r}
u_{j-1}-u_{j}=\frac{G_{0} \delta_{x}^{2}}{2 \nu}+\left[2\left(j-j_{0}\right)-1\right] \cdot \frac{F \delta_{x}^{2}}{2 \nu} \\
\text { for } j \geq j_{0}+3 .
\end{array}
$$

Equation (B4) at $j=j_{0}+h / 2$ and at $j=j_{0}+h / 2+1$ gives

$$
\begin{aligned}
& u_{j_{0}+h / 2-1}-u_{j_{0}+h / 2}=\frac{G_{0} \delta_{x}^{2}}{2 \nu}+(h-1) \frac{F \delta_{x}^{2}}{2 \nu}, \\
& u_{j_{0}+h / 2}-u_{j_{0}+h / 2+1}=\frac{G_{0} \delta_{x}^{2}}{2 \nu}+(h+1) \frac{F \delta_{x}^{2}}{2 \nu} .
\end{aligned}
$$

Adding Eqs. (B5) and (B6) yields

$$
u_{j_{0}+h / 2-1}-u_{j_{0}+h / 2+1}=\frac{G_{0} \delta_{x}^{2}}{\nu}+\frac{F h \delta_{x}^{2}}{\nu} .
$$

Since the velocity of the Poiseuille flow takes its maximum value at the central plane $j=j_{0}+h / 2$, the velocity adjacent to the central plane has the same value, as follows:

$$
u_{j_{0}+h / 2-1}=u_{j_{0}+h / 2+1}
$$


The substitution of Eq. (B8) into Eq. (B7) gives $G_{0}=$ $-F h$. The theoretical velocity profile $\bar{u}$ for a Poiseuille flow is given by

$$
\bar{u}=-\frac{F \delta_{x}^{2}}{2 \nu}\left(j-j_{0}\right)\left(j-j_{0}-h\right)
$$

Since $u_{j_{0}+2}=\bar{u}_{j_{0}+2}$ beyond the IBL, Eq. (B9) yields

$$
\bar{u}_{j_{0}}-u_{j_{0}+2}=-\frac{F \delta_{x}^{2}(h-2)}{\nu} .
$$

From Eqs. (B2), (B3), and (B10), we obtain Eq. (67) for the numerical velocity slip.

Equations (9) and (B2) yield

$$
\begin{array}{r}
u_{b}=\frac{1}{2}\left(u_{j_{0}}+u_{j_{0}+1}\right) \\
=u_{j_{0}}+\frac{\delta_{x}^{2}}{\nu}\left(\frac{G_{0}(\phi-1)}{8}-\frac{F}{4}\right) .
\end{array}
$$

Since $u^{d}=u_{b}$ in the implicit correction method, Eq. (B11) gives Eq. (69). For the direct forcing method, Eqs. (8) and (B11) give Eq. (70).
[1] C. S. Peskin, J. Comput. Phys. 10, 252 (1972).

[2] S. Chen and G. D. Doolen, Annu. Rev. Fluid Mech. 30, 329 (1988).

[3] Z. G. Feng and E. E. Michaelides, J. Comput. Phys. 195, 602 (2004).

[4] Z. G. Feng and E. E. Michaelides, J. Comput. Phys. 202, $20(2005)$.

[5] X. D. Niu, C. Shu, Y. T. Chew, and Y. Peng, Phys. Lett. A 354, 173 (2006).

[6] J. Wu and C. Shu, J. Comput. Phys. 228, 1963 (2009).

[7] H. K. Jeong, H. S. Yoon, M. Y. Ha, and M. Tsutahara, J. Comput. Phys. 229, 2526 (2010).

[8] T. Seta, Phys. Rev. E 87, 063304 (2013).

[9] G. Le and J. Zhang, Phys. Rev. E 79, 026701 (2009).

[10] I. Ginzburg, F. Verhaeghe, and D. d'Humières, Commun. Comput. Phys. 3, 427 (2008).

[11] K. Hayashi, R. Rojas, T. Seta, and A. Tomiyama, J. Comput. Multiphase Flows 4, 193 (2012).

[12] I. Ginzburg, D. d'Humières, and A. Kuzmin, J. Stat. Phys. 139, 1090 (2010).

[13] P. Lallemand and L. S. Luo, Phys. Rev. E 61, 6546 (2000).

[14] J. Lu, H. Han, B. Shi, and Z. Guo, Phys. Rev. E 85,
016711 (2012).

[15] X. He, Q. Zou, L. S. Luo, and M. Dembo, J. Stat. Phys. 87, 115 (1997).

[16] M. Uhlmann, J. Comput. Phys. 209, 448 (2005).

[17] Z. Guo, C. Zheng, and B. Shi, Phys. Rev. E 65, 046308 (2002).

[18] S. K. Kang and Y. A. Hassan, Int. J. Numer. Meth. Fluids 66, 1132 (2011).

[19] I. Ginzburg, Commun. Comput. Phys. 11, 1439 (2012).

[20] X. Yang, X. Zhang, Z. Li, and G. W. He, J. Comput. Phys. 228, 7821 (2009).

[21] S. C. R. Dennis and G. Z. Chang, J. Fluid Mech. 42, 471 (1970).

[22] F. Nieuwstadt and H. B. Keller, Comput. Fluids 1, 59 (1973).

[23] X. He and G. Doolen, J. Comput. Phys. 134, 306 (1997).

[24] D. d'Humières, I. Ginzburg, M. Krafczyk, P. Lallemand, and L. S. Luo, Philos. Trans. R. Soc. Lond. A 360, 437 (2002).

[25] Y. Peng, C. Shu, Y. T. Chew, X. D. Niu, and X. Y. Lu, J. Comput. Phys. 218, 460 (2006). 